\title{
A Rigorous Block Spin Approach to Massless Lattice Theories $^{\star}$
}

\author{
K. Gawedzki ${ }^{1}$ and A. Kupiainen ${ }^{2}$ \\ Lyman Laboratory of Physics, Harvard University, Cambridge, Massachusetts 02138, USA
}

\begin{abstract}
The renormalization group technique is used to study rigorously the $\lambda(\nabla \phi)^{4}$ perturbation of the massless lattice field $\phi$ in dimensions $d \geqq 2$. Asymptoticity of the perturbation expansion in powers of $\lambda$ is established for the free energy density. This is achieved by using Kadanoff's block spin transformation successively to integrate out high momentum degrees of freedom and by applying ideas previously used by Gallavotti and Balaban in the context of the ultraviolet problems. The method works for arbitrary semibounded polynomials in $\nabla \phi$ and $\Delta \phi$.
\end{abstract}

\section{Introduction}

During the last fifteen years renormalization group $(\mathrm{RG})$ has become one of the main tools in both quantum field theory and statistical mechanics. Still, compared to the numerous works devoted to a variety of heuristic applications of this method, see e.g. the articles [1] and references therein, the rigorous results concerning $\mathrm{RG}$ are rather few, dealing with very simple models or attempting a rigorous formulation of the problems [2]. Here an exceptional position is occupied by the work of Gallavotti and collaborators [3, 4], where RG ideas were employed as a tool to prove ultraviolet stability of $\phi_{3}^{4}$ quantum field theory. This allowed to turn RG into a powerful method in rigorous study of more complicated superrenormalizable field theory models, see [5] for an announcement of results concerning gauge theories.

In this paper we modify the technique developed by Gallavotti et al. and apply it to an infrared problem, namely the lattice model with hamiltonian density $\frac{1}{2}(\nabla \phi)^{2}+\lambda(\nabla \phi)^{4}$, in dimensions $d \geqq 2$. The method works for arbitrary "irrelevant"

* Supported in part by the National Science Foundation under Grant No. PHY 79-16812

1 On leave from Department of Mathematical Methods of Physics, University, PL-00-682 Warsaw, Poland

2 Address after Sept. 1, 1980: Helsinki University, Research Institute for Theoretical Physics, SF-00170 Helsinki 17, Finland 
(with respect to the Gaussian fixed point) polynomials involving $\nabla \phi$ and $\Delta \phi$. For simplicity we consider only the least irrelevant case. Our modification goes along the lines suggested by Balaban [5] who proposed to use Kadanoff's block spin transformation version of RG in the context of the ultraviolet problem. The main result of the present paper is establishing of asymptoticity of the perturbation series in powers of $\lambda$ for the free energy of the model mentioned above. We hope to be able to obtain also information about correlations and their decay using similar ideas and to extend the results to more complicated models, such as the dipole gas, in the future.

To understand why the standard techniques which would work if we added a mass term $\frac{1}{2} m^{2} \phi^{2}$ to the hamiltonian fail here, write the free energy in unit volume (pressure) $p(\lambda)$ as

$$
p(\lambda)=\lim _{\Lambda \uparrow \mathbb{Z}^{d}} \frac{1}{|\Lambda|} \log \int \exp \left[-\lambda \sum_{x \in \Lambda}\left(\nabla \phi_{x}\right)^{4}\right] d \mu_{G}(\phi),
$$

where $d \mu_{G}$ is the lattice Gaussian measure with covariance $G=(-\Delta)^{-1}$ (which makes perfect sense in $d \geqq 3$ ). We may generate the perturbation expansion for $p(\lambda)$, together with the remainder, by writing (formally)

$$
\begin{aligned}
p(\lambda) & =\lim _{\Lambda \uparrow \mathbb{Z}^{d}} \frac{1}{|\Lambda|} \int_{0}^{1} d t \frac{d}{d t} \log \int \exp \left[-\lambda \sum_{x}\left(\nabla \phi_{x}\right)^{4}\right] d \mu_{G}(\phi) \\
& =\int_{0}^{1} d t\left\langle-\lambda\left(\nabla \phi_{x_{0}}\right)^{4}\right\rangle_{t \lambda},
\end{aligned}
$$

where

$$
\langle\cdot\rangle_{t \lambda}=\int \cdot \exp \left[-t \lambda \sum_{x}\left(\nabla \phi_{x}\right)^{4}\right] d \mu_{G}(\phi) / \int \exp \left[-t \lambda \sum_{x}\left(\nabla \phi_{x}\right)^{4}\right] d \mu_{G}(\phi),
$$

and subsequently integrating by parts using the formula

$$
\int \nabla \phi_{x_{0}} F(\nabla \phi) d \mu_{G}(\phi)=\sum_{x_{1}}\left(\nabla \nabla G_{x_{0} x_{1}}\right) \int \frac{\delta F(\nabla \phi)}{\delta \nabla \phi_{x_{1}}} d \mu_{G}(\phi) .
$$

Performing (1.3) sufficiently many times we can generate from (1.1) the perturbation series to arbitrary order $\tau$ together with the remainder, which is a sum of terms of the form (we suppress indices in $\nabla_{\mu}$ )

$$
C_{\tau} \lambda^{\tau+1} \sum_{x_{1}, \ldots, x_{n}} \prod_{\left(i_{\alpha}, j_{\alpha}\right)}\left(\nabla \nabla G_{x_{\tau_{\alpha}} x_{j_{\alpha}}}\right) \int_{0}^{1} d t\left\langle\prod_{i=0}^{n}\left(\nabla \phi_{x_{t}}\right)^{n_{t}}\right\rangle_{t \lambda}
$$

with lines $\left(i_{\alpha}, j_{\alpha}\right)$ forming a connected graph joining all points $x_{0}, x_{1}, \ldots, x_{n}$. Then, assuming one could bound the expectations in (1.4) (e.g. using superstability of the interaction), summability of the propagators

$$
\sum_{x_{1}}\left|\nabla \nabla G_{x_{0} x_{1}}\right|<\infty
$$

would imply finiteness of the remainder and hence asymptoticity of the perturbation expansion for the pressure. The problem, however, is that (1.5), which is obvious in the case of massive free propagator decaying exponentially fails for 
massless $G$ where, although $\sum_{x_{1}} \nabla \nabla G_{x_{0} x_{1}}$ exists it does not converge absolutely since $\left|\nabla \nabla G_{x_{0} x_{1}}\right|=O\left(\left|x_{0}-x_{1}\right|^{-d}\right)$ for large $\left|x_{0}-x_{1}\right|$. It should be remarked that this gives rise to no infrared divergences in the perturbation series which is finite as one easily infers through momentum space analysis, but invalidates the above argument which provided an estimate for the remainder.

The idea we will follow in this paper is to save the argument by applying it to the integral over the high momentum degrees of freedom (rather than to the total integration at once) and then repeating the procedure many times. The renormalization group transformation we use here is Kadanoff's block spin transformation and it consists of two steps: integration over fluctuations within square blocks of $3^{d}$ spins, keeping the average spins in the blocks fixed, and rescaling the distance by $1 / 3$ and the spin variables according to their canonical dimension, i.e. by the factor $3^{(d-2) / 2}$. Hence, the first block spin transformation may be written as

$$
\int \delta\left(\phi^{\prime}-C \phi\right) e^{-\lambda \sum_{x \in \Lambda}\left(\nabla \phi_{x}\right)^{4}} d \mu_{G}(\phi) \equiv e^{V_{1}\left(\phi^{\prime}\right)} d \mu_{G_{1}}\left(\phi^{\prime}\right),
$$

where

$$
(C \phi)_{y}=3^{\frac{d-2}{2}} \frac{1}{3^{d}} \sum_{r_{\mu}=0, \pm 1} \phi_{3 y+r}
$$

gives the (rescaled) block spins. $V_{1}\left(\phi^{\prime}\right)$ is the effective interaction for the new distance scale (the old one multiplied by three), and $G_{1}$ is the new covariance (defined as the one which would appear if $\lambda=0$ ).

(1.6) is now iterated yielding effective hamiltonians $V_{n}$, describing the system in scale $3^{n}$, and covariances $G_{n}$. The point is that formally the transformation drives our measure to a Gaussian fixed point. Namely, the canonical dimension of our coupling (in units of length) is $d$. Thus, heuristically one expects the effective coupling for distance scale $3^{n}$ to be $\sim 3^{-n d} \lambda$ and thus $V_{n}$ to go to zero. This is not quite, but almost, what happens. In fact, $V_{n}$ will approximately have the form

$$
\begin{aligned}
V_{n}(\phi) \cong & (\phi-\text { independent term })-O(\lambda) \sum_{x \in 3^{-n_{\Lambda}}}\left(\nabla \phi_{x}\right)^{2} \\
& -3^{-n d} \lambda \sum_{x \in \mathcal{3}^{-n_{\Lambda}}}\left(\nabla \phi_{x}\right)^{4}+\text { "smaller" terms }
\end{aligned}
$$

and the covariances $G_{n}$ tend to a massless Gaussian fixed point

$$
\left(G_{\infty}\right)_{x y}=\int_{\Delta(x)} d \bar{x} \int_{\Delta(y)} d \bar{y}\left(-\Delta_{c}\right)^{-1}(\bar{x}, \bar{y})
$$

where $\Delta(x)$ is the unit cube centered at $x \in \mathbb{Z}^{d}$ and $\Delta_{c}$ is the continuum Laplace operator. $G_{\infty}$ has long distance behavior identical to that of $(-\Delta)^{-1}$. Note that $(\nabla \phi)^{4}$ in (1.8) has the expected $3^{-n d}$ factor (thus being "irrelevant") but we also obtained a quadratic ("marginal") term which does not disappear as $n \rightarrow \infty$. This corresponds to the fact that there is a continuum of Gaussian fixed points for Kadanoff's transformation, namely $\mu G_{m} \mu>0$, and the one corresponding to our 
interaction with $\lambda \neq 0$ is different from the $\lambda=0$ case. The transformation thus generates terms which take us to the correct fixed point, $(1+0(\lambda)) G_{\infty}$.

In practice it is convenient to perform the block spin transformation by realizing the Gaussian measure $d \mu_{G_{n}}$ as a product of two measures, one corresponding to the block spin degrees of freedom and the other to the fluctuations within the blocks. Then the block spin transformation consists simply of integration with respect to the second measure. This brings our formulation quite close to the setting of [3].

The problem with implementing the above procedure is, of course, that formally $V_{n}$ is an infinite series of arbitrary many body interactions and in order to obtain the perturbation series for $p(\lambda)$ up to order $\lambda^{\tau}$ we want to retain at least all the terms of order less than or equal to $\tau$, and preferably to be able to ignore the rest. However, it is not obvious that we can do that since the spins $\phi$ are unbounded. Here we apply the ideas of Gallavotti et al. which allow to eliminate large gradients due to their small probability assured by positivity properties of the effective hamiltonians. Now we can compute $V_{n}$ perturbatively to order $\tau$ by integrating by parts with respect to the fluctuation measure as in (1.1)-(1.4). The remainder can be estimated since the fluctuation covariance has exponential falloff (the high momentum cutoff provides an effective mass) uniformly in $n$ and the external fields (block spins) are bounded. The bound for the remainder will be $O\left(3^{-n d} \lambda^{\tau+1}\right), 3^{-n d}$ coming from contraction of interaction volume in subsequent effective hamiltonians. Summing over $n$ would then give the $O\left(\lambda^{\tau+1}\right)$ bound for difference between $p(\lambda)$ and its perturbation expansion up to order $\tau$, thus proving asymptoticity of the latter.

Actually slight problems arise from the marginal terms in $V_{n}$. Namely those of order higher than 2 eventually pick $\log$ (cutoff) $\sim n$ contributions due to mainly technical reasons. These would eventually spoil the positivity properties of $V_{n}$ and we have to stop the iteration when $n \lambda^{2} \sim \lambda$ i.e. $n^{-1} \sim \lambda$. However, then our interaction lives in the volume $3^{-n d}|\Lambda| \sim 3^{-d / \lambda}|\Lambda|$ and it turns out that a brute force argument suffices to complete the estimation.

The paper is organized as follows: After Introduction, Chap. 2 gives a detailed description of the model and of the block spin transformations and states the main results. Chapter 3 establishes a lower (easier) bound for the difference between $p(\lambda)$ and its perturbation expansion to order $\tau$ and Chap. 4 the (more involved) upper bound - they are separated because the arguments used to eliminate large spin variables are different in both cases. Detailed properties of effective hamiltonians used in the estimation are proven in Chap. 5. Finally, Chap. 6 contains proofs of probability estimates used to eliminate large spins. We would like to stress the fact that the technical core of our estimates is based on combining integration by parts with Ruelle's superstability estimates [6]. This distinguishes it from the technical part of [3] which dealt with continuum fields and used in an essential way the Markov property of the measures, lacking here, and hard results of the theory of elliptic boundary value problems [4].

After this work was finished we obtained a paper by J. Bricmont, J. Fontaine, J. Lebowitz, and T. Spencer proving asymptoticity of the perturbation expansion for pressure and also for correlation functions in the same model by a different method. 


\section{Block Spin Transformation}

In this chapter we define our model, formulate more precisely the main result and discuss some properties of the block spin transformation.

We work with periodic boundary conditions. Let $\Lambda$ denote the set of integral points in the periodic box $\left[-\frac{3^{N}}{2}, \frac{3^{N}}{2}\right]^{d}, d \geqq 2$. This will be our initial lattice. We shall also use lattices $\Lambda_{n}$ consisting of integral points in the periodic boxes $\left[-\frac{3^{N-n}}{2}, \frac{3^{N-n}}{2}\right]^{d}, n=0,1, \ldots, N$, and $\Lambda^{n}:=3^{-n} \Lambda$. Notice that $\Lambda=\Lambda_{0}=\Lambda^{0}$. Points in $\Lambda_{n}$ will be denoted by letters $x, y, u, v, \ldots$, and those in $\Lambda^{n}$ by $z$. For any two points in one of these lattices the magnitude of their difference with subscript $p$ will denote their periodic distance.

Let $\Delta$ denote the lattice laplacean on $\Lambda$, i.e. $(\phi,-\Delta \phi)=\sum_{\substack{x, y \in A \\|x, y|_{p}=1}}\left(\phi_{x}-\phi_{y}\right)^{2} \cdot \Delta$ has a zero mode and we shall define the massless Gaussian process $\phi$ on $\Lambda$ as that with covariance $G_{0}$ inverting $-\Delta$ on the subspace orthogonal to the zero mode. More precisely,

where

$$
\left(G_{0}\right)_{x y}=\frac{1}{|\Lambda|} \sum_{\substack{0 \neq p \in \frac{2 \pi}{3^{N}} \mathbb{Z}^{d} \\\left|p_{\kappa}\right| \leqq \pi}} \mu(p)^{-1} e^{i p(x-y)},
$$

$$
\mu(p)=2 \sum_{\kappa}\left(1-\cos p_{\kappa}\right) .
$$

Let $d \mu_{G_{0}}$ denote the measure of the process. It is concentrated on $\phi$ 's orthogonal to the zero mode. Denote the lattice gradient by $\nabla$ and consider the random process $\left(\nabla \phi_{x}\right)^{4}$, where we use shorthand notation $\left(\nabla \phi_{x}\right)^{2 m} \equiv\left(\sum_{\mu}\left(\nabla_{\mu} \phi_{x}\right)^{2}\right)^{m}$. The periodic free energy density (pressure) $p(\lambda)$ in volume $\Lambda$ is defined for $\lambda \geqq 0$ by

$$
p(\lambda):=\frac{1}{|\Lambda|} \log \int \exp \left[-\lambda \sum_{x \in \Lambda}\left(\nabla \phi_{x}\right)^{4}\right] d \mu_{G_{0}}(\phi) .
$$

Our main result consists of showing that the perturbation expansion for $p(\lambda)$ in powers of $\lambda$ is asymptotic, uniformly in $\Lambda$. This yields asymptoticity of the perturbation expansion for the infinite volume free energy density in the thermodynamic limit. Throughout the paper we shall always assume $\lambda$ to be bounded from above.

It is convenient to define for $D \subset A$

$$
\begin{aligned}
V_{0}^{D}:= & -\lambda \sum_{x \in D}\left(\nabla \phi_{x}\right)^{4}-\sum_{k=1}^{\tau} \frac{(-\lambda)^{k}}{k !} \sum_{x_{1}, \ldots, x_{k} \in D} \\
& \cdot\left\langle\left(\nabla \phi_{x_{1}}\right)^{4} ; \ldots ;\left(\nabla \phi_{x_{k}}\right)^{4}\right\rangle_{G_{0}}^{T},
\end{aligned}
$$

where $\langle\ldots\rangle_{G_{0}}^{T}$ denotes the truncated expectations with respect to $d \mu_{G_{0}}$. The truncated expectations on the right hand side of (2.3) give the first $\tau$ orders of 
perturbation expansion for the free energy in volume $D$. Now our main result may be restated as

Theorem 2.1. For each $\tau$ and for small $\lambda$

$$
\left|\frac{1}{|\Lambda|} \log \int \exp V_{0}^{\Lambda} d \mu_{G_{0}}\right|=O\left(\lambda^{\tau+1}\right)
$$

uniformly in $\Lambda$.

Remark. (2.4) will be deduced from two bounds

$$
e^{-O\left(\lambda^{\tau+1 / 2}\right)|\Lambda|} \leqq \int \exp V_{0}^{\Lambda} d \mu_{G_{0}} \leqq e^{O\left(\lambda^{\tau+1 / 2}\right)|\Lambda|}
$$

proven to hold uniformly in $|\Lambda|$ in Chaps. 3 and 4. Of course (2.5) yields immediately (2.4) with " $=O\left(\lambda^{\tau+1}\right)$ " replaced by " $\leqq O\left(\lambda^{\tau+1 / 2}\right)$ ". However, the latter with $\tau$ higher by one implies (2.4) in its original form. From now on we shall consider $\tau$ fixed.

As stressed in the Introduction, in the proof of (2.4) a critical role is played by Kadanoff's block spin transformation [7]. We shall describe it in detail now.

The block spin variables are defined exactly as in the Introduction and the first block spin transformation consists of integration $\left(\phi \equiv \phi^{0}\right)$

$$
\int \delta\left(\phi^{1}-C \phi^{0}\right) \exp \left[V_{0}^{\Lambda}\left(\phi^{0}\right)\right] d \mu_{G_{0}}\left(\phi^{0}\right)
$$

with $C$ given by (1.7) but now transforming finite dimensional spaces $\mathbb{R}^{\Lambda} \rightarrow \mathbb{R}^{\Lambda_{1}}$. In the case when $\lambda=0(2.6)$ is easily computable yielding

Lemma 2.1.

$$
d \phi_{1} \int \delta\left(\phi^{1}-C \phi^{0}\right) d \mu_{G_{0}}(\phi)=d \mu_{G_{1}}\left(\phi^{1}\right),
$$

where

$$
G_{1}:=C G_{0} C^{T}
$$

$\left(C^{T}\right.$ denotes the transposed operation mapping $\mathbb{R}^{\Lambda_{1}} \rightarrow \mathbb{R}^{\Lambda}$ ).

Proof. Immediate, since

$$
\begin{aligned}
\int e^{i\left(\phi^{1}, h\right)} d \phi^{1} \int \delta\left(\phi^{1}-C \phi^{0}\right) d \mu_{G_{0}}\left(\phi^{0}\right) & =\int e^{i\left(C \phi^{0}, h\right)} d \mu_{G_{0}}\left(\phi^{0}\right) \\
& =\exp \left[-\frac{1}{2}\left(h, C G_{0} C^{T} h\right)\right],
\end{aligned}
$$

where $(\cdot, \cdot)$ denotes the scalar product.

Denote by $E$ the projection on the constant sequences in $\mathbb{R}^{\Lambda_{n}}$. Explicit computation, see (A.1), shows that $E \mathbb{R}^{\Lambda_{1}}$ is the zero eigen-subspace for $G_{1}$ and that $G_{1}$ is invertible on $(I-E) \mathbb{R}^{\Lambda_{1}}$. With a slight abuse of notation define $G_{1}^{-1}$ to be the inverse of $G_{1}$ on $(I-E) \mathbb{R}^{\Lambda_{1}}$ and to annihilate $E \mathbb{R}^{\Lambda_{1}}$. Notice that

$$
G_{1} G_{1}^{-1}=I-E \text {. }
$$

We shall compute (2.6) by perturbation expansion around the $\lambda=0$ case (2.7). To this end it is convenient to realize the Gaussian process $\phi$ as a sum of two independent ones, corresponding to block spin variables and fluctuations inside blocks respectively. 
Let $\phi^{1}$ be the Gaussian process indexed by the points of $\Lambda_{1}$ with covariance $G_{1}$. Let us also consider another process, $\xi^{0}$, living on $\bar{\Lambda}:=\Lambda-3 \Lambda_{1}$, that is on points of $\Lambda$ which are not centers of the $3 \times \ldots \times 3$ blocks. $\xi^{0}$ will have the covariance

$$
\Gamma_{0}:=R\left(G_{0}-G_{0} C^{T} G_{1}^{-1} C G_{0}\right) R^{T},
$$

where

$$
R: \mathbb{R}^{4} \rightarrow \mathbb{R}^{\bar{A}}
$$

is the restriction.

It is shown in Appendix (see Proposition A.3) that $\Gamma_{0}$ is a strictly positive operator on $\mathbb{R}^{\bar{A}}$. Let now $A_{0}: \mathbb{R}^{\Lambda_{1}} \rightarrow \mathbb{R}^{\Lambda}$ be given by

$$
A_{0}:=G_{0} C^{T} G_{1}^{-1}
$$

and $Q: \mathbb{R}^{\bar{A}} \rightarrow \mathbb{R}$ by

We have

$$
(Q \xi)_{x}=\left\{\begin{array}{lll}
\xi_{x} & \text { if } \quad x \in \bar{\Lambda} \\
-\sum_{\substack{r: \\
r_{\kappa}=0, \pm 1}} \xi_{x+r} & \text { if } \quad x \in \Lambda \backslash \bar{\Lambda} .
\end{array}\right.
$$

Lemma 2.2. The process $\phi^{0}$ may be written in terms of the two independent processes $\phi^{1}$ and $\xi^{0}$ as

$$
\phi^{0}=A_{0} \phi^{1}+Q \xi^{0} .
$$

Proof. We have to show that the covariance of $A_{0} \phi^{1}+Q \xi^{0}$, which is $A_{0} G_{1} A_{0}^{T}$ $+Q \Gamma_{0} Q^{T}$, equals $G_{0}$. By (2.10) and (2.13)

$$
A_{0} G_{1} A_{0}^{T}=G_{0} C^{T}(1-E) G_{1}^{-1} C G_{0}=G_{0} C^{T} G_{1}^{-1} C G_{0} .
$$

On the other hand by (2.11)

$$
Q \Gamma_{0} Q^{T}=Q R\left(G_{0}-G_{0} C^{T} G_{1}^{-1} C G_{0}\right) R^{T} Q^{T} .
$$

Now $G_{0}-G_{0} C^{T} G_{1}^{-1} C G_{0}$ maps on sequences in $\mathbb{R}^{A}$ with vanishing averages over the blocks since by (2.8) and (2.10)

$$
C\left(G_{0}-G_{0} C^{T} G_{1}^{-1} C G_{0}\right)=C G_{0}-(I-E) C G_{0}=E C G_{0}=C E G_{0}=0
$$

as $E$ is the zero mode projection of $G_{0}$. But on sequences with zero-block-averages $Q R$ acts as identity. Hence

$$
Q \Gamma_{0} Q^{T}=\left(G_{0}-G_{0} C^{T} G_{1}^{-1} C G_{0}\right) R^{T} Q^{T}=G_{0}-G_{0} C^{T} G_{1}^{-1} C G_{0} .
$$

(2.16) and (2.19) prove our assertion.

To see that the decomposition (2.15) realizes the splitting of the process $\phi_{0}$ into the block-spin and the fluctuation parts insert it to (2.6). This gives

$$
\begin{aligned}
(2.6) & =\int \delta\left(\phi^{1}-C\left(A_{0} \bar{\phi}^{1}+Q \xi^{0}\right)\right) \exp \left[V^{A}\left(A_{0} \bar{\phi}^{1}+Q \xi^{0}\right)\right] d \mu_{G_{1}}\left(\bar{\phi}^{1}\right) d \mu_{\Gamma_{0}}\left(\xi^{0}\right) \\
& =\int \delta\left(\phi^{1}-(I-E) \bar{\phi}^{1}\right) \exp \left[V^{A}\left(A_{0} \bar{\phi}^{1}+Q \xi^{0}\right)\right] d \mu_{G_{1}}\left(\bar{\phi}^{1}\right) d \mu_{\Gamma_{0}}\left(\xi^{0}\right) \\
& =d \mu_{G_{1}}\left(\phi^{1}\right) \int \exp \left[V^{A}\left(A_{0} \phi^{1}+Q \xi^{0}\right)\right] d \mu_{\Gamma_{0}}\left(\xi^{0}\right),
\end{aligned}
$$


where we have used

$$
C A_{0}=I-E, \quad C Q=0
$$

and the fact that $d \mu_{G_{1}}\left(\bar{\phi}^{1}\right)$ is concentrated on $\bar{\phi}^{1}$ such that $E \bar{\phi}^{1}=0$ since $E$ is the zero eigen-projection of $G_{1}$. Thus in the language of realization (2.15) of $\phi^{0}$ the block spin transformation consists of integrating out $\xi^{0}$. Let $F \in L^{1}\left(d \mu_{G_{0}}\right)$. Define $T_{1}: L^{1}\left(d \mu_{G_{0}}\right) \rightarrow L^{1}\left(d \mu_{G_{1}}\right)$ by

$$
\left(T_{1} F\right)\left(\phi^{1}\right)=\int F\left(A_{0} \phi^{1}+Q \xi^{0}\right) d \mu_{\Gamma_{0}}\left(\xi^{0}\right) .
$$

We have an obvious formula

$$
\int F d \mu_{G_{0}}=\int T_{1} F d \mu_{G_{1}} .
$$

$T_{1}$ gives the first block spin transformation. The next ones will be defined similarly by induction. Put

$$
\phi^{m-1}=A_{m-1} \phi^{m}+Q \xi^{m-1}
$$

where

$$
A_{m-1}=G_{m-1} C^{T} G_{m}^{-1}
$$

with $G_{m}$, the covariance of $\phi^{m}$, being

$$
G_{m}=C G_{m-1} C^{T}=C^{m} G_{0}\left(C^{T}\right)^{m}
$$

and

$$
G_{m} G_{m}^{-1}=I-E
$$

$\xi^{m}$ has covariance

$$
\Gamma_{m-1}=R\left(G_{m-1}-G_{m-1} C^{T} G_{m}^{-1} C G_{m-1}\right) R^{T} .
$$

$\phi^{m}$ is indexed by the points of $\Lambda_{m}$ and $\xi^{m}$ by those of $\bar{\Lambda}_{m} \equiv \Lambda_{m}-3 \Lambda_{m+1}$. We get analogues of (2.22) and (2.23)

$$
\begin{aligned}
\left(T_{m} F\right)\left(\phi^{m}\right) & =\int F\left(A_{m-1} \phi^{m}+Q \xi^{m-1}\right) d \mu_{\Gamma_{m-1}}\left(\xi^{m-1}\right), \\
\int F d \mu_{G_{m-1}} & =\int T_{m} F d \mu_{G_{m}} .
\end{aligned}
$$

Certain $F$ 's are reproduced in their form under $T_{m}$. Introduce a kernel $\mathscr{A}_{m z y}$ for $z \in \Lambda^{m}$ and $y \in \Lambda_{m}$ (see the beginning of this chapter for the notation)

$$
\mathscr{A}_{m z y}:=3^{\left(\frac{d-2}{2}\right) m}\left(A A_{1} \ldots A_{m-1}\right)_{3 m_{z, y}} .
$$

Put

$$
\psi^{m}:=\mathscr{A}_{m} \phi^{m}, \quad \zeta^{m}:=\mathscr{A}_{m} Q \xi^{m}
$$

so that the random processes $\psi^{m}$ and $\zeta^{m}$ are indexed by the points of $\Lambda^{m}$. As we shall show in Appendix relations (2.32) are "almost local". With

$$
\nabla_{\mu} \psi_{z}^{m} \equiv 3^{m}\left(\psi_{z+3-m_{e_{\mu}}}-\psi_{z}^{m}\right)
$$


and $\int_{D} d z$ denoting the Riemann sum $3^{-m d} \sum_{z \in D}$ for $D \subset \Lambda^{m}$, consider the general local Wick monomials of degree $M$ in $\nabla \psi^{m}$ :

$$
P_{\mu_{1} \ldots \mu_{M}}^{m}:=\int_{\Lambda^{m}}: \prod_{i=1}^{M} \nabla_{\mu_{i}} \psi_{z}^{m}:_{G_{m}} d z
$$

\section{Lemma 2.3}

$$
T_{m} P_{\mu_{1} \ldots \mu_{M}}^{m-1}=3^{-d\left(\frac{M}{2}-1\right)} P_{\mu_{1} \ldots \mu_{M}}^{m} .
$$

Proof. By (2.24), (2.31), and (2.32)

$$
\psi_{z}^{m-1}=3^{-\frac{d-2}{2}} \psi_{z / 3}^{m}+\zeta_{z}^{m-1}
$$

and consequently

$$
\nabla \psi_{z}^{m-1}=3^{-\frac{d}{2}} \nabla \psi_{z / 3}^{m}+\nabla \zeta_{z}^{m-1}
$$

Since the Gaussian processes $\nabla_{\mu} \psi_{z}^{m}$ and $\nabla_{v} \zeta_{z}^{m-1}$ are independent, we have

$$
: \prod_{i=1}^{M} \nabla_{\mu_{i}} \psi_{z}^{m-1}:_{G_{m-1}}=\sum_{I \subset\{1, \ldots, M\}}: \prod_{i \notin I} 3^{-\frac{d}{2}} \nabla_{\mu_{i}} \psi_{z / 3}^{m}:_{G_{m}}: \prod_{i \in I} \nabla_{\mu_{i}} \zeta_{z}^{m-1}:_{\Gamma_{m-1}} .
$$

Under the $d \mu_{\Gamma_{m-1}}$ expectation all terms but the one with $I=\emptyset$ vanish. Hence

$$
\begin{aligned}
T_{m} P_{\mu_{1} \ldots \mu_{M}}^{m-1} & =\int P_{\mu_{1} \ldots \mu_{M}}^{m-1} d \mu_{\Gamma_{m-1}}\left(\zeta^{m-1}\right) \\
& =\int_{\Lambda^{m-1}}: \prod_{i=1}^{M}\left(3^{-\frac{d}{2}} \nabla_{\mu_{\imath}} \psi_{z / 3}^{m}\right):_{G_{m}} d z=3^{-d\left(\frac{M}{2}-1\right)} P_{\mu_{1} \ldots \mu_{M}}^{m} .
\end{aligned}
$$

\section{Corollary 2.4}

$$
T_{n} T_{n-1} \ldots T_{1} P_{\mu_{1} \ldots \mu_{M}}^{0}=3^{-d\left(\frac{M}{2}-1\right) n} P_{\mu_{1} \ldots \mu_{M}}^{n} .
$$

If the terms of the type $P_{\mu_{1} \ldots \mu_{M}}^{0}$ appear in the initial hamiltonian then in the first order approximation the block spin transformations reproduce their form, as (2.35) and (2.39) show, except for an overall factor $3^{-d(M / 2-1) n}$. This is driven to zero for $M>2$ (irrelevant hamiltonians) and stays constant if $M=2$ (marginal ones). Thus it is natural to write our hamiltonian in volume $D$ given by (2.3) in Wick ordered form

$$
\begin{aligned}
V_{0}^{D}= & -\lambda \sum_{\mu \nu} M_{\mu \nu} \int_{D}: \nabla_{\mu} \psi_{z}^{0} \nabla_{\nu} \psi_{z}^{0}:_{G_{0}} d z-\lambda \int_{D}:\left(\nabla \psi_{z}^{0}\right)^{4}:_{G_{0}} d z \\
& \text { + field-independent polynomial in } \lambda \text { of order } \tau \\
& \text { starting with a } \lambda^{2} \text { term, } \\
M_{\mu \nu}= & 2 \delta_{\mu \nu} \sum_{\kappa} \int \nabla_{\kappa} \phi_{x}^{0} \nabla_{\kappa} \phi_{x}^{0} d \mu_{G_{0}}+4 \int \nabla_{\mu} \phi_{x}^{0} \nabla_{v} \phi_{x}^{0} d \mu_{G_{0}} \\
= & 2 \delta_{\mu \nu}\left(1-|\Lambda|^{-1}\right)+4 \int \nabla_{\mu} \phi_{x}^{0} \nabla_{\nu} \phi_{x}^{0} d \mu_{G_{0}} .
\end{aligned}
$$

The first term on the right hand side of (2.40) is marginal, the second irrelevant according to the above analysis. However, the information about how the 
hamiltonian behaves under the linearized renormalization group transformation is not sufficient. We shall have to trace the effect of the transformation beyond the first order of perturbation expansion, in fact up to order $\tau$. To this end define inductively for $D \subset A^{m}$

$$
V_{m}^{D}:=\left.\sum_{k=0}^{\tau} \frac{\lambda^{k}}{k !} \frac{d^{k}}{d \lambda^{k}}\right|_{\lambda=0} \log T_{m} \exp V_{m-1}^{3 D}
$$

Iterating (2.42) gives

$$
V_{n}^{D}=\left.\sum_{k=0}^{\tau} \frac{\lambda^{k}}{k !} \frac{d^{k}}{d \lambda^{k}}\right|_{\lambda=0} \log T_{n} T_{n-1} \ldots T_{1} \exp V_{0}^{3^{n} D}
$$

Notice that $V_{N}^{D}=0$, where $N$ defines the volume $\Lambda$.

By (2.29)

$$
\begin{gathered}
\left(T_{n} T_{n-1} \ldots T_{1} \exp V_{0}^{3^{n} D}\right)\left(\phi^{n}\right)=\int \exp \left[V _ { 0 } ^ { 3 ^ { n } D } \left(A_{0} \ldots A_{n-1} \phi^{n}\right.\right. \\
\left.\left.+A_{0} \ldots A_{n-2} Q \xi^{n-1}+\ldots+Q \xi^{0}\right)\right] d \mu_{\Gamma_{n-1}}\left(\xi^{n-1}\right) \ldots d \mu_{\Gamma_{0}}\left(\xi^{0}\right) .
\end{gathered}
$$

Thus by cumulant expansions formula

$$
V_{n}^{D}=\sum_{k=1}^{\tau} \frac{1}{k !}\left\langle V_{0}^{3^{n} D} ; \ldots ; V_{0}^{3^{n} D}\right\rangle_{\substack{n \\ m=0}}^{T} \Gamma_{m}
$$

where $\langle\ldots\rangle_{\oplus \Gamma_{m}}^{T}$ denotes the truncated expectations with respect to $d \mu_{\Gamma_{n-1}}\left(\xi^{n-1}\right) \cdot \ldots \cdot d \mu_{\Gamma_{0}}\left(\xi^{0}\right)$. To compute the right hand side of $(2.45)$ it is convenient to use the random fields $\psi^{n}$ and $\zeta^{n-1}, \ldots, \zeta^{0}[$ see (2.32)]. Iteration of (2.37) gives

$$
\nabla_{\mu} \psi_{z}^{0}=3^{-\frac{d}{2} n} \nabla_{\mu} \psi_{\frac{z}{3^{n}}}^{n}+3^{-\frac{d}{2}(n-1)} \nabla_{\mu} \zeta_{\frac{z}{3^{n-1}}}^{n-1}+\ldots+3^{-\frac{d}{2}} \nabla_{\mu} \zeta_{z}+\nabla_{\mu} \zeta_{z}^{0}
$$

Notice that the fields on the right hand side are independent. Together with $(2.40)$ and (2.45), (2.46) allows an effective computation of $V_{n}^{D}$ by use of Wick's theorem. The results, after the change of variables $z \rightarrow 3^{n} z$, may be expressed in terms of (generally non-local) Wick-ordered polynomials in random variables $\nabla \psi_{z}^{m}$. We shall divide these polynomials into three groups writing

$$
V_{n}^{D}=V_{n, 0}^{D}+V_{n, 1}^{D}+V_{n, 2}^{D} \text {. }
$$

$V_{n, 0}^{D}$ includes the terms that are of the first order in $\lambda$ :

$$
V_{n, 0}^{D}=-\lambda \sum_{\mu, v} M_{\mu v} \int_{D}: \nabla_{\mu} \psi_{z}^{n} \nabla_{v} \psi_{z}^{n}:_{G_{n}} d z-3^{-d n} \lambda \int_{D}:\left(\nabla \psi_{z}^{n}\right)^{4}:_{G_{n}} d z
$$

Note that (2.48) is identical in form with the first two terms of the initial hamiltonian (2.40) except for the factor $3^{-d n}$ at the quartic term. This is in agreement with the previous analysis of the linearized renormalization group transformation given in Corollary 2.4 .

$V_{n, 1}^{D}$ groups all contributions to $V_{n}^{D}-V_{n, 0}^{D}$ which are Wick-ordered monomials of non-zero order in $\nabla \psi$ 's and $V_{n, 2}^{D}$ all $\nabla \psi$-independent terms. A diagramatic analysis gives the following result: 
Proposition 2.5. $V_{n, 1}^{D}+V_{n, 2}^{D}$ may be written as a finite sum of terms

$$
\int_{D} \ldots \int_{D} I_{\left(\mu_{\alpha}^{1}\right) \ldots\left(\mu_{\alpha}^{r}\right)}\left(z_{1}, \ldots, z_{r}\right): \prod_{\varrho=1}^{r} \prod_{\alpha=1}^{a_{\varrho}} \nabla_{\mu_{\alpha}} \psi_{z_{\varrho}}^{n}:_{G_{n}} d z_{1} \ldots d z_{r}
$$

with $2 \leqq r \leqq \tau, 0 \leqq a_{\varrho} \leqq 3$ and D-independent I's satisfying for each $1 \leqq \varrho_{0} \leqq r$ the bound

$$
\int_{\Lambda^{n}} \ldots \int_{\Lambda^{n}}\left|I_{\left(\mu_{\alpha}^{1}\right) \ldots\left(\mu_{\alpha}^{r}\right)}\left(z_{1}, \ldots, z_{r}\right)\right| \prod_{\varrho \neq \varrho_{0}} d z_{\varrho} \leqq \lambda^{r} C\left(1+n^{C}\right) .
$$

Moreover, for I's corresponding to terms contributing to $V_{n, 1}^{D}$, i.e. such that $\sum_{\varrho=1}^{r} \alpha_{\varrho}>0$, (2.50) holds also with $I \ldots\left(z_{1}, \ldots, z_{r}\right)$ replaced by $I \ldots\left(z_{1}, \ldots, z_{r}\right) \exp \left[\varepsilon d\left(z_{1}, \ldots, z_{r}\right)\right]$ for small $\varepsilon>0$, where $d\left(z_{1}, \ldots, z_{r}\right)$ is the length of the shortest connected graph joining the points $\left(z_{1}, \ldots, z_{r}\right)$ in the periodic lattice $\Lambda^{m}$.

In Proposition 2.5 as throughout the whole paper $C$ and $\varepsilon$ denote various constants which are independent of $\lambda, \Lambda$, and $n$. They do not have to stand for the same constants even when they appear several times in the same formula.

The proof of Proposition 2.5 will be deferred until Chap. 5. Here let us mention that the main input are the estimates giving a uniform exponential decay of $\Gamma_{m x x^{\prime}}$ and resulting decays for $\left\langle\nabla \zeta_{z}^{m} \nabla \zeta_{z^{\prime}}^{m}\right\rangle_{\Gamma_{m}}$ and $\left\langle\nabla \psi_{z}^{m} \nabla \psi_{z^{\prime}}^{m}\right\rangle_{G_{n}}$ (exponential and polynomial ones respectively) proven in Appendix, Proposition A.7 and A.8.

\section{The Lower Bound}

In this chapter we shall prove the first (lower) bound of (2.5). This will be done inductively by inserting suitable characteristic functions under the integral and integrating out the fluctuation field. The characteristic functions will cut off large values of the fields $\nabla \psi^{n}$ and $\nabla \zeta^{n}$ in a $\lambda$ dependent way: the smaller $\lambda$ the higher the cut-off. Let

$$
B=b \log \left(1+\lambda^{-1}\right),
$$

where $b$, independent of $n, \lambda$, and $\Lambda$, will be chosen later. Denote

$$
\chi_{B}\left(\nabla \psi^{n}\right) \equiv \prod_{z \in \Lambda^{n}} \chi\left(\left\|\nabla \psi_{z}^{n}\right\| \leqq B\right)
$$

Let for $n=0,1, \ldots, N-1$

$$
\begin{aligned}
& Z_{n}=\int \chi_{B}\left(\nabla \psi^{n}\right) \exp V_{n}^{\Lambda^{n}} d \mu_{G_{n}} \\
& Z_{n}^{\prime}=\int \chi_{B}\left(\nabla \psi^{n}\right) d \mu_{G_{n}} \\
& Z_{N}=Z_{N}^{\prime}=1(N \text { defines the volume } \Lambda)
\end{aligned}
$$

We shall prove

Proposition 3.1. There exists $\delta>0$ such that for $\lambda$ small enough and $n=0,1, \ldots, \min \left(\left[\lambda^{-\delta}\right]-1, N-1\right)$

$$
Z_{n} \geqq \exp \left[-3^{-d n} C \lambda^{\tau+1 / 2}|\Lambda|\right] Z_{n+1} .
$$


Given Proposition 3.1, we can prove the lower bound easily. Iteration of (3.3) gives

$$
Z_{0} \geqq \exp \left[-C \lambda^{\tau+1 / 2}|\Lambda|\right] Z_{n(\lambda, \Lambda)+1},
$$

where $n(\lambda, \Lambda):=\min \left(\left[\lambda^{-\delta}\right]-1, N-1\right)$. If $n(\lambda, \Lambda)=N-1$ we are done since $Z_{N}=1$. Suppose that $n(\lambda, \Lambda)=\left[\lambda^{-\delta}\right]-1$. Using (2.47)-(2.50) of Proposition 2.5 we obtain the bound

$$
\left|V_{n}^{\Lambda^{n}}\right| \leqq C \lambda\left(1+n^{C}\right) B^{C}\left|\Lambda^{n}\right| \quad \text { if } \quad\left\|\nabla \psi_{z}^{n}\right\| \leqq B
$$

(the Wick-ordering of the $\nabla \psi^{n}$ monomials causes no trouble since $\left\langle\nabla \psi_{z}^{n} \nabla \psi_{z^{\prime}}^{n}\right\rangle_{G_{n}}$ is uniformly bounded, see Proposition A.8). Thus,

$$
\left|V_{n}^{\Lambda^{n}}\right|_{n=[\lambda-\delta} \leqq C \lambda\left(1+\left(\left[\lambda^{-\delta}\right]-1\right)^{C}\right) B^{C} 3^{-d \lambda-\delta}|\Lambda| \leqq C \lambda^{\tau+1 / 2}|\Lambda| .
$$

Now we may replace $Z_{n(\lambda, \Lambda)+1}$ on the right hand side of (3.4) by $Z_{n(\lambda, \Lambda)+1}^{\prime}$ increasing $C$ and continue iterating (3.3) in the $Z^{\prime}$ version until $n=N-1$ when it yields the lower bound (2.5).

Proof of Proposition 3.1. First let us notice that in order to get rid of large values of $\left\|\nabla \psi^{n}\right\|$ it is enough to restrict both $\left\|\nabla \psi^{n+1}\right\|$ and $\left\|\nabla \zeta^{n}\right\|$. Namely (2.37) gives

in the obvious notation.

$$
\chi_{B}\left(\nabla \psi^{n}\right) \geqq \chi_{B}\left(\nabla \psi^{n+1}\right) \chi_{\frac{2}{3} B}\left(\nabla \zeta^{n}\right)
$$

Moreover, since by (2.32)

$$
\nabla \zeta^{n}=\nabla \mathscr{A}_{n} Q \xi^{n}
$$

and the kernels $\mathscr{A}_{n z x}$ have a uniform (in $\Lambda$ and $n$ ) exponential decay, as shown in Proposition A.6, in order to restrict $\nabla \zeta^{n}$ it is enough to bound $\xi^{n}$ :

$$
\chi_{\frac{2}{3} B}\left(\nabla \zeta^{n}\right) \geqq \chi_{\bar{B}}\left(\xi^{n}\right),
$$

where $\bar{B}=\gamma B$ with $\gamma$ independent of $\lambda, \Lambda, n$. (2.37), (3.7), and (3.8) together with (3.2) allow to write

$$
Z_{n} \geqq \int \chi_{B}\left(\nabla \psi^{n+1}\right)\left(\int \chi_{\bar{B}}\left(\xi^{n}\right) \exp V_{n}^{A^{n}} d \mu_{\Gamma_{n}}\right) d \mu_{G_{n+1}} .
$$

To extract from the $d \mu_{\Gamma_{n}}$ integral the desired $\exp V_{n+1}^{A^{n+1}}$ term, the integration-byparts argument sketched in Introduction is applied. We write

$$
\begin{aligned}
\int \chi_{\bar{B}} \exp V_{n}^{A^{n}} d \mu_{\Gamma_{n}} & =\exp \left[\int_{0}^{1} d t \frac{d}{d t} \log \int \chi_{\bar{B}} \exp \left(t V_{n}^{\Lambda^{n}}\right) d \mu_{\Gamma_{n}}\right] \cdot \int \chi_{\bar{B}} d \mu_{\Gamma_{n}} \\
& =\exp \left[\int_{0}^{1} d t\left\langle V_{n}^{\Lambda^{n}}\right\rangle_{t}\right] \int \chi_{\bar{B}} d \mu_{\Gamma_{n}},
\end{aligned}
$$

where

$$
\langle\cdot\rangle_{t} \equiv \int \cdot \chi_{\bar{B}} \exp \left(t V_{n}^{\Lambda^{n}}\right) d \mu_{\Gamma_{n}} \iint \chi_{\bar{B}} \exp \left(t V_{n}^{\Lambda^{n}}\right) d \mu_{\Gamma_{n}} .
$$

The integration by parts is performed according to the formula

$$
\left\langle\xi_{x}^{n} F\left(\xi^{n}\right)\right\rangle_{t}=\sum_{y \in \bar{A}_{n}} \Gamma_{n x y}\left\langle\left(\frac{\delta}{\delta \xi_{y}^{n}}+t \frac{\delta V_{n}^{A}}{\delta \xi_{y}^{n}}+\frac{\delta \log \chi_{\bar{B}}}{\delta \xi_{y}^{n}}\right) F\left(\xi^{n}\right)\right\rangle_{t}
$$


where the $\frac{\delta \log \chi_{\bar{B}}}{\delta \xi_{y}^{n}}$ term should be understood as replacing the characteristic function $\chi\left(\left|\xi_{y}^{n}\right| \leqq \bar{B}\right)$ in the numerator of $\langle\cdot\rangle_{t}$ by the sum of $\delta$-functions $\frac{\delta \chi\left(\left|\xi_{y}^{n}\right| \leqq \bar{B}\right)}{\delta \xi_{y}^{n}}$.

Since $V_{n}^{\Lambda^{n}}$ is a polynomial in the $\xi^{n}$ field we may apply (3.12) to $\left\langle V_{n}^{\Lambda^{n}}\right\rangle_{t}$ or to any polynomial-like term produced by the earlier integrations by parts. The $\xi^{n}$-independent terms, the terms with $\frac{\delta \log \chi_{\bar{B}}}{\delta \xi_{y}^{n}}$ or the ones with the overall power of $\lambda$ larger than $\tau$ will not be transformed further. After a finite number of steps all terms are of this form. Then we may write

$$
\log \int \chi_{\bar{B}} \exp V_{n}^{\Lambda} d \mu_{\Gamma_{n}}-\log \int \chi_{\bar{B}} d \mu_{\Gamma_{n}} \equiv \int_{0}^{1} d t\left\langle V_{n}^{\Lambda}\right\rangle_{t}=V_{n+1}^{\Lambda^{n+1}}+\int d t\left\langle R_{n}(t)\right\rangle_{t},
$$

where $V_{n+1}^{\Lambda^{n+1}}$ groups all $\xi^{n}$-independent terms of order $\leqq \tau$ in $\lambda$ and $R_{n}(t)$ all terms either of order $>\tau$ in $\lambda$ or with $\frac{\delta \log \chi_{\bar{B}}}{\delta \xi_{y}^{n}}$ contributions. That the $\xi^{n}$-independent terms reproduce exactly $V_{n+1}^{A^{n+1}}$ is easily seen from the inductive definition (2.42).

The structure of $R_{n}$ follows from Proposition 2.5 and the method to generate it. First write the $\xi^{n}$ dependent part of $V_{n}^{A}$ as a combination of terms

$$
\lambda^{r} \sum_{x_{1}, \ldots, x_{s} \in \bar{A}_{n}} j\left(x_{1}, \ldots, x_{s} ; \nabla \psi^{n+1}\right) \prod_{\sigma=1}^{s} \xi_{x_{\sigma}}^{n}
$$

undoing the Wick ordering in (2.48), (2.49) and using (2.37) and (2.32). Due to the uniform exponential clustering of $\nabla \mathscr{A}_{n z x^{\prime}}$ see Proposition A.6, and Proposition 2.5 j's satisfy

$$
\sum_{\substack{x_{\sigma \in \bar{\Lambda}_{n}} \\ \sigma \neq \sigma_{0}}}\left|j\left(x_{1}, \ldots, x_{s} ; \nabla \psi^{n+1}\right)\right| \leqq C\left(1+n^{C}\right)\left(1+\left\|\nabla \psi^{n+1}\right\|_{L^{\infty}}^{C}\right) .
$$

Now the integration by parts either

A) contracts $\xi_{x_{\sigma}}^{n}$ with $\xi_{x_{\sigma}}^{n}$ replacing it by $\Gamma_{n x_{\sigma} x_{\sigma}}$ within one or between two expressions (3.14) or

B) contracts $\xi_{x_{\sigma}}^{n}$ of (3.14) to $\frac{\delta \log \chi_{\bar{B}}}{\delta \xi_{y}^{n}}$.

In case A) we produce either another term of the type (3.14) or a $\xi^{n}$-independent term

$$
\lambda^{r} j\left(\nabla \psi^{n+1}\right)
$$

with

$$
\left|j\left(\nabla \psi^{n+1}\right)\right| \leqq C\left(1+n^{C}\right)\left(1+\left\|\nabla \psi^{n+1}\right\|_{L^{\infty}}^{C}\right)\left|\Lambda_{n}\right|
$$

as easily follows from the uniform exponential decay of $\Gamma_{n}$ 's. In case $B$ we obtain a term

$$
\lambda^{r} \sum_{x_{1}, \ldots, x_{s} \in \bar{A}_{n}} j\left(x_{1}, \ldots, x_{s-1}, x_{s} ; \nabla \psi^{n+1}\right) \prod_{\sigma=1}^{s-1} \xi_{x_{\sigma}}^{n} \frac{\delta \log \chi_{\bar{B}}}{\delta \xi_{x_{s}}^{n}}
$$

again with $j$ satisfying (3.15). 
Summarizing, $R_{n}(t)$ is a combination of terms (3.14) and (3.16) with $r \geqq \tau+1$ and of terms (3.18). Since on the support of $\chi_{B}\left(\nabla \psi^{n+1}\right) \chi_{B}\left(\xi^{n}\right)$

$$
\left\|\nabla \psi^{n+1}\right\|_{L^{\infty}} \leqq B \text { and }\left\|\xi^{n}\right\|_{L^{\infty}} \leqq \bar{B} \equiv \gamma B
$$

we obtain finally using the bounds (3.15) and (3.17)

$$
\left|\int_{0}^{1} d t\left\langle R_{n}(t)\right\rangle_{t}\right| \leqq C\left(1+n^{C}\right) B^{C}\left|\Lambda_{n}\right|\left(\lambda^{\tau+1}+\sup \left\langle\left|\frac{\delta \log \chi_{\bar{B}}}{\delta \xi_{x}^{n}}\right|\right\rangle_{t}\right),
$$

where "sup" on the right hand side is over $\nabla \psi^{n+1}$ with $\left\|\nabla \psi^{n+1}\right\|_{L^{\infty}} \leqq B, x \in \bar{\Lambda}_{n}$ and $t \in[0,1]$.

To complete the proof of Proposition 3.1 we need the following estimates which express the fact that probability of large values of the $\xi^{n}$ fields is small. They will be proven in Chap. 6 together with their counterparts used in the proof of the upper bound.

Lemma 3.2. Provided that $b$ in (3.1) is large enough and $\lambda$ is small enough

$$
\sup \left\langle\left|\frac{\delta \log \chi_{\bar{B}}}{\delta \xi_{x}^{n}}\right|\right\rangle_{t} \leqq e^{-c \bar{B}^{2}}
$$

and

$$
\int \chi_{\bar{B}} d \mu_{\Gamma_{n}} \geqq e^{-C e^{-C \bar{B}^{2}\left|\bar{\Lambda}_{n}\right|}} .
$$

Using (3.20) together with $e^{-C \bar{B}^{2}} \leqq C \lambda^{\tau+1}$ we get from (3.19)

$$
\left|\int_{0}^{1} d t\left\langle R_{n}(t)\right\rangle_{t}\right| \leqq 3^{-d n} C\left(1+n^{C}\right) B^{C} \lambda^{\tau+1}|\Lambda| \leqq 3^{-d n} C \lambda^{\tau+1 / 2}|\Lambda|
$$

for $n \leqq \lambda^{-\delta}$ if $\delta$ is chosen so that $\lambda^{1 / 2}\left(1+\lambda^{-\delta C}\right) B^{C} \leqq 1$. Now (3.9), (3.10), (3.13), (3.21), and (3.22) give

$$
\begin{aligned}
Z_{n} & \geqq Z_{n+1} \exp \left[-3^{-d n} C \lambda^{\tau+1 / 2}|\Lambda|\right] e^{-C e^{-C \overline{\boldsymbol{B}}^{2}\left|\bar{\Lambda}_{n}\right|}} \\
& \geqq Z_{n+1} \exp \left[-3^{-d n} C \lambda^{\tau+1 / 2}|\Lambda|\right]
\end{aligned}
$$

which is (3.3).

The version of (3.3) with $Z$ replaced by $Z^{\prime}$ follows from (3.21) above.

\section{The Upper Bound}

In proving the second inequality of (2.5) (the upper bound) we shall use a slightly different argument, also borrowed from [3], to eliminate the large values of the fields. Namely, we shall exclude the interaction from the regions where the fields are large. To this end define

$$
D_{n}:=\left\{z \in \Lambda^{n}:\left\|\nabla \psi_{z}^{n}\right\| \geqq B\right\}, \quad D_{n}^{\prime}:=\left\{z \in \Lambda^{n}:\left\|\nabla \psi_{z}^{n}\right\| \geqq \frac{1}{2} 3^{-\frac{d}{2}} B\right\},
$$

where $B$ is the $\lambda$-dependent constant given by (3.1) with $b$ large enough.

The manipulations with the interaction region will be based on the following estimates using the positivity of the leading terms of the interaction hamiltonians. 
Lemma 4.1. Let $b$ of (3.1) be large enough.

$$
V_{0}^{\Lambda} \leqq V_{0}^{\Lambda \backslash D_{0}}
$$

Moreover, there exists $\delta>0$ such that for small $\lambda, n=0,1, \ldots, \min \left(\left[\lambda^{-\delta}\right], N-1\right)$ and $B_{n} \subset D_{n}^{c}$

$$
\begin{aligned}
& V_{n}^{B_{n}} \leqq V_{n}^{B_{n} \backslash C_{n}} \quad \text { if } \quad C_{n} \subset D_{n}^{\prime} .
\end{aligned}
$$

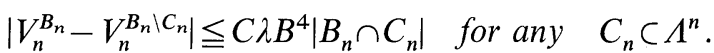

Proof. A) First notice that the difference of the field-independent terms in (2.40) for $D=\Lambda$ and $D=\Lambda \backslash D_{0}$ is by virtue of Proposition 2.5 a combination of the terms

$$
\int_{\Lambda} \ldots \int_{\Lambda} \int_{D_{0}} \int_{\Lambda \backslash D_{0}} \ldots \int_{\Lambda \backslash D_{0}} I\left(z_{1}, \ldots, z_{r}\right) d z_{1} \ldots d z_{r}
$$

and, as a result of (2.50), is bounded by $\lambda^{2} C\left|D_{0}\right|$. However, the difference of the first two terms in (2.40) for $D=\Lambda$ and $D=\Lambda \backslash D_{0}$ is easily seen to be less than $-\lambda C B^{4}\left|D_{0}\right|$ if only $b$ in (3.1) is chosen large enough so that the negative quartic term dominates. terms

B) Similarly as in A) $\left(V_{n, 1}^{B_{n}}+V_{n, 2}^{B_{n}}\right)-\left(V_{n, 1}^{B_{n} \backslash C_{n}}+V_{n, 2}^{B_{n} \backslash C_{n}}\right)$ is a combination of the

$$
\int_{B_{n}} \ldots \int_{B_{n}} \int_{B_{n} \cap C_{n}} \int_{B_{n} \backslash C_{n}} \ldots \int_{B_{n} \backslash C_{n}} I\left(z_{1}, \ldots, z_{r}\right): \prod_{\varrho=1}^{r}\left(\nabla \psi_{z_{e}}^{n}\right)^{a_{\varrho}}:_{G_{n}} d z_{1} \ldots d z_{r}
$$

[where we suppressed the $\mu$ subscripts of (2.49)] and is bounded by $C \lambda^{2}\left(1+n^{C}\right) B^{C}\left|B_{n} \cap C_{n}\right|$ because of (2.50) and the bound $\left\|\nabla \psi^{n}\right\| \leqq B$ holding on $D_{n}^{c}$.

Now in $V_{n, 0}^{B_{n}}-V_{n, 0}^{B_{n} \backslash C_{n}}$ the quartic term is negative if $b$ in (3.1) is large enough but it is suppressed by the $3^{-d n}$ factor. However, the quadratic factor is also negative $\left[M_{\mu \nu}\right.$ is positive definite, see (2.41)] and is bounded above by $-C \lambda B^{2}\left|B_{n} \cap C_{n}\right|$ for $b$ large. This dominates $C \lambda^{2}\left(1+n^{C}\right) B^{C}\left|B_{n} \cap C_{n}\right|$ if $n \geqq \lambda^{-\delta}$ for small $\delta$ and if $\lambda$ is small enough.

C) This is proven as $B$ except for the term $V_{n, 0}^{B_{n}}-V_{n, 0}^{B_{n} \backslash C_{n}}$ whose magnitude is bounded by $C \lambda\left(B^{2}+3^{-d n} B^{4}\right)\left|B_{n} \cap C_{n}\right|$ which dominates again the $C \lambda^{2}\left(1+n^{C}\right) B^{C}\left|B_{n} \cap C_{n}\right|$ contribution yielding (4.4).

Remark 4.2. For proving (4.3) we have used the negativity of the marginal quadratic term of $V_{n}^{D}$ to dominate the other irrelevant terms. In other $(\nabla \psi)^{2 n}$ models the quadratic term coming from the Wick ordering does not have to be negative. However, we may extract a small negative $O(\lambda)$ quadratic term from $d \mu_{G_{0}}$ and use it to the same end.

Define now

$$
Z^{n}:=\int \exp V_{n}^{\Lambda^{n} \backslash D_{n}} d \mu_{G_{n}}
$$

for $n=0,1, \ldots, N-1$ and

$$
Z^{N}:=1 .
$$

Here is the upper-bound counterpart of Proposition 3.1. 
Proposition 4.3. For $\delta>0$ small enough, $\lambda$ small enough and $n=0,1, \ldots, \min \left(\left[\lambda^{-\delta}\right]-1, \mathrm{~N}-1\right)$

$$
Z^{n} \leqq \exp \left[3^{-d n} C \lambda^{\tau+1 / 2}|\Lambda|\right] Z^{n+1} \text {. }
$$

Now, since by Lemma $4.1 \mathrm{~A}$

$$
\int \exp V_{0}^{\Lambda} d \mu_{G_{0}} \leqq Z^{0}
$$

the iteration of (4.7) gives

$$
\int \exp V_{0}^{\Lambda} d \mu_{G_{0}} \leqq \exp \left[C \lambda^{\tau+1 / 2}|\Lambda|\right] Z^{n(\lambda, \Lambda)+1}
$$

similarly as in the proof of the lower bound, compare (3.4). If $n(\lambda, \Lambda)=N-1$ we are done again. In the other case

$$
\begin{aligned}
\left.Z^{[\lambda-\delta}\right] & \leqq \int \exp \left[V_{n}^{\left.\left.\Lambda^{n} \backslash D_{n}\right|_{n=[\lambda-\delta}\right] d \mu_{G_{n}}}\right. \\
& \leqq\left.\exp \left[C \lambda B^{4}\left|\Lambda^{n} \backslash D_{n}\right|\right]\right|_{n=[\lambda-\delta]} \leqq \exp \left[C \lambda B^{4} 3^{-d \lambda-\delta}|\Lambda|\right] \\
& \leqq \exp \left[C \lambda^{\tau+1 / 2}|\Lambda|\right],
\end{aligned}
$$

where we have used Lemma 4.1C.(4.8) and (4.9) give the upper bound (2.5).

Proof of Proposition 4.3. We start by inserting a partition of unity to $Z^{n}$ in order to control the range of the values of $\xi^{n}$ at each point. Denote by $\bar{p}$ a collection of even integers $\left\{p_{x}\right\}_{x \in \bar{\Lambda}_{n}}$. Let $\chi_{p_{x}}\left(\xi_{x}^{n}\right)$ be the following characteristic functions

$$
\chi_{p_{x}}\left(\xi_{x}^{n}\right):=\chi\left(\xi_{x}^{n} \in\left[\left(p_{x}-1\right) \bar{B},\left(p_{x}+1\right) \bar{B}\right)\right)
$$

where $\bar{B}=\gamma B$ and $\gamma$ will be specified in a moment. We denote also

Given $\bar{p}$, define

$$
\chi_{\bar{p}}\left(\xi^{n}\right) \equiv \prod_{x \in \bar{\Lambda}_{n}} \chi_{p_{x}}\left(\xi_{x}^{n}\right)
$$

$$
R_{n}=\bigcup_{x \in \Lambda_{n}}\left\{z \in \Lambda^{n}:|z-x|_{p}<b^{\prime} B^{2} \log \left(1+\left|p_{x}\right|\right)\right.
$$

We have

$$
\begin{aligned}
Z^{n} & =\sum_{\bar{p}} \int\left(\int \chi_{\bar{p}} \exp V_{n}^{\Lambda^{n} \backslash D_{n}} d \mu_{\Gamma_{n}}\right) d \mu_{G_{n+1}} \\
& \leqq \sum_{\bar{p}} \int\left(\int \chi_{\bar{p}} \exp V_{n}^{\Lambda^{n} \backslash\left(D_{n} \cup R_{n}\right)} d \mu_{\Gamma_{n}}\right) d \mu_{G_{n+1}} \exp \left[C \lambda B^{4}\left|R_{n}\right|\right],
\end{aligned}
$$

where we have used Lemma 4.1C.

The purpose of the elimination of the interaction from $R_{n}$ becomes clear because of the following

Lemma 4.4. If $\gamma$ in the definition of $\bar{B}$ is small enough and $b^{\prime}$ in (4.11) is large enough then

$$
\left\|\nabla \zeta_{z}^{n}\right\| \leqq \frac{1}{2} 3^{-1 / 2} B \text { for } z \text { in } R_{n}^{c} .
$$

Proof. By virtue of (2.32) and of the uniform exponential decay of $\nabla \mathscr{A}_{n z x^{\prime}}$ see Proposition A.3,

$$
\left\|\nabla \zeta_{z}^{n}\right\| \leqq \sum_{x: p_{x} \neq 0} C \bar{B} e^{-\varepsilon|z-x|_{p}}\left(1+\left|p_{x}\right|\right)+C \bar{B}
$$


But if $p_{x} \neq 0$ then, since $z \in R_{n}^{c},|z-x|_{p} \geqq b^{\prime} B^{2} \log \left(1+\left|p_{x}\right|\right)$. Hence

$$
\sum_{x: p_{x} \neq 0} e^{-\left.\varepsilon\right|_{z}-\left.x\right|_{p}}\left(1+\left|p_{x}\right|\right) \leqq \sum_{x} \exp \left[-\varepsilon|z-x|_{p}+\frac{1}{b^{\prime} B^{2}}|z-x|_{p}\right] \leqq C
$$

if $b^{\prime}$ in (4.11) is large enough. (4.14) and (4.15) yield (4.13) if $\gamma$ is small enough.

The next step in estimation of $Z^{n}$ will be to remove the interaction on the right hand side of (4.12) from the region $3 D_{n+1}$. Notice that on $R_{n}^{c} \cap 3 D_{n+1}$

$$
\left\|\nabla \psi_{z}^{n}\right\| \geqq 3^{-\frac{d}{2}}\left\|\nabla \psi_{z / 3}^{n+1}\right\|-\left\|\nabla \zeta_{z}^{n}\right\| \geqq 3^{-\frac{d}{2}} B-\frac{1}{2} 3^{-\frac{d}{2}} B=\frac{1}{2} 3^{-\frac{d}{2}} B
$$

by virtue of (2.37) and Lemma 4.4, so that $R_{n}^{c} \cap 3 D_{n+1} \subset D_{n}^{\prime}$. Hence we may use Lemma 4.1B in order to replace in (4.12) $V_{n}^{\Lambda^{n} \backslash\left(D_{n} \cup R_{n}\right)}$ by $V_{n}^{\Lambda^{n} \backslash\left(D_{n} \cup 3 D_{n+1} \cup R_{n}\right)}$.

Moreover

$$
D_{n} \subset R_{n} \cup 3 D_{n+1}
$$

or equivalently

$$
R_{n}^{c} \cap 3 D_{n+1}^{c} \subset D_{n}^{c}
$$

again by (2.37) and Lemma 4.4. Hence we obtain

$$
Z^{n} \leqq \sum_{\bar{p}} \int\left(\int \chi_{\bar{p}} \exp V_{n}^{A^{n} \backslash\left(3 D_{n+1} \cup R_{n}\right)} d \mu_{T_{n}}\right) d \mu_{G_{n+1}} \exp \left[C \lambda B^{4}\left|R_{n}\right|\right] .
$$

Notice that the interaction region $\Lambda^{n} \backslash\left(3 D_{n+1} \cup R_{n}\right) \equiv \tilde{\Lambda}^{n}$ in (4.17) does not depend any more on $\xi^{n}$ 's over which we integrate in the $n^{\prime}$ th step of the block spin transformation. As in the proof of Proposition 3.1 we compute this integral using integration by parts. Write

$$
\int \chi_{\bar{p}} \exp V_{n}^{\tilde{A}^{n}} d \mu_{\Gamma_{n}}=\exp \left[\int_{0}^{1} d t\left\langle V_{n}^{\tilde{\Lambda}^{n}}\right\rangle_{\bar{p}, t}\right] \int \chi_{\bar{p}} d \mu_{\Gamma_{n}},
$$

compare (3.10). As in Chap. 3

$$
\int_{0}^{1} d t\left\langle V_{n}^{\tilde{A}^{n}}\right\rangle_{\bar{p}, t}=V_{n+1}^{3-1 \tilde{A}^{n}}+\int_{0}^{1} d t\left\langle R_{n}(t)\right\rangle_{\bar{p}, t}
$$

where $R_{n}(t)$ is again a combination of the terms (3.14), (3.16) with $n \geqq \tau+1$ and (3.18) (with $\log \chi_{\bar{p}}$ replacing $\log \chi_{\bar{B}}$ ). Now j's satisfy a modified version of (3.15) and (3.17). The modification replaces $j$ in (3.15) by $j \exp \left[\varepsilon \sum_{\sigma=1}^{s} d\left(x_{\sigma}, \tilde{\Lambda}^{n}\right)\right]$ and $\left\|\nabla \psi^{n+1}\right\|_{L^{\infty}}$ in (3.15) and (3.17) by $\left\|\nabla \psi^{n+1}\right\|_{L^{\infty}\left(\tilde{A}^{n}\right)}$. This is clearly possible since $\xi^{n}$ enters $V_{n}^{\tilde{X}^{n}}$ only via $\nabla \zeta^{n} \Gamma_{\Lambda^{n}}$ to which it is connected by an exponentially decaying kernel, and $\nabla \psi^{n+1}$ only via $\nabla \psi^{n+1} \uparrow_{\tilde{\Lambda}^{n}}$. Notice that since large values of $\left|\xi_{x}^{n}\right|$ may appear only for $x$ far from $\tilde{\Lambda}_{n}$,

$$
\begin{aligned}
e^{-\varepsilon d\left(x, \tilde{A}^{n}\right)}\left|\xi_{x}\right| & \leqq e^{-\varepsilon d\left(x, R_{n}^{c}\right)}\left|\xi_{x}\right| \\
& \leqq e^{-\varepsilon d\left(x, R_{n}^{c}\right)} \bar{B}\left(1+\left|p_{x}\right|\right) \\
& \leqq e^{-\varepsilon b^{\prime} B^{2} \log \left(1+\left|p_{x}\right|\right)}\left(1+\left|p_{x}\right|\right) \\
& \leqq C
\end{aligned}
$$


if $b^{\prime}$ in (4.11) is large enough. Also

$$
\left\|\nabla \psi^{n+1}\right\|_{L^{\infty}\left(\tilde{\Lambda}^{n}\right)} \leqq B
$$

Hence,

$$
\left|\int_{0}^{1} d t\left\langle R_{n}(t)\right\rangle_{t, \bar{p}}\right| \leqq C\left(1+n^{C}\right) B^{C}\left|\Lambda_{n}\right|\left(\lambda^{\tau+1}+\sup e^{-\varepsilon d\left(x, \tilde{\Lambda}^{n}\right)}\left\langle\left|\frac{\delta \log \chi_{\bar{p}}}{\delta \xi_{x}^{n}}\right|\right\rangle_{\bar{p}, t}\right),
$$

compare (3.19).

We need the following probability estimate which will be proven in Chap. 6 :

Lemma 4.5. Provided that $b$ in (3.1) and $b^{\prime}$ in (4.11) are large enough and $\lambda$ is small enough,

$$
\sup e^{-\varepsilon d\left(x, \tilde{\Lambda}^{n}\right)}\left\langle\left|\frac{\delta \log \chi_{p}}{\delta \xi_{x}^{n}}\right|\right\rangle_{\bar{p}, t} \leqq e^{-C \bar{B}^{2}}
$$

and

$$
\int \chi_{\bar{p}}\left(\xi^{n}\right) d \mu_{\Gamma_{n}} \leqq \exp \left[-C \bar{B}^{2} \sum_{x} p_{x}^{2}\right] .
$$

(4.20) and (4.21) give

$$
\left|\int_{0}^{1} d t\left\langle R_{n}(t)\right\rangle_{\bar{p}, t}\right| \leqq 3^{-d n} C \lambda^{\tau+1 / 2}|\Lambda|,
$$

compare (3.22). (4.17)-(4.19) and (4.23) yield in turn

$$
Z^{n} \leqq \sum_{\bar{p}} \int \exp V_{n+1}^{3-1 \tilde{\Lambda}^{n}} d \mu_{G_{n+1}} \cdot \int \chi_{\bar{p}} d \mu_{\Gamma_{n}} \cdot \exp \left[\lambda B^{4}\left|R_{n}\right|+3^{-d n} C \lambda^{\tau+1 / 2}|\Lambda|\right]
$$

Now $3^{-1} \tilde{\Lambda}^{n}=\Lambda^{n+1} \backslash\left(D_{n+1} \cup 3^{-1} R_{n}\right)$ and we may restore the interaction in $\left(\Lambda^{n+1} \backslash D_{n+1}\right) \cap 3^{-1} R_{n}$ in order to obtain $Z^{n+1}$ using again Lemma 4.1C. The sum over $\bar{p}$ is controlled with help of (4.22) which shows that non-zero values of $\bar{p}$ have very small probability. Thus,

$$
\begin{aligned}
Z^{n} \leqq & Z^{n+1} \sum_{\bar{p}} \int \chi_{\bar{p}} d \mu_{\Gamma_{n}} \cdot \exp \left[C \lambda B^{4}\left|R_{n}\right|+3^{-d n} C \lambda^{\tau+1 / 2}|\Lambda|\right] \\
\leqq & Z^{n+1} \sum_{\bar{p}} \exp \left[\sum_{x \in \bar{\Lambda}_{n}}\left(\lambda B^{4}\left(2 b^{\prime} B^{2} \log \left(1+\left|p_{x}\right|\right)\right)^{d}-C \bar{B}^{2} p_{x}^{2}\right)\right] \\
& \cdot \exp \left[3^{-d n} C \lambda^{\tau+1 / 2}|\Lambda|\right] \\
\leqq & Z^{n+1} \sum_{\bar{p}} \exp \left[-\sum_{x} C \bar{B}^{2} p_{x}^{2}\right] \exp \left[3^{-d n} C \lambda^{\tau+1 / 2}|\Lambda|\right] \\
\leqq & Z^{n+1} \exp \left[e^{-C \bar{B}^{2}}\left|\bar{\Lambda}_{n}\right|+3^{-d n} C \lambda^{\tau+1 / 2}|\Lambda|\right] \\
\leqq & Z^{n+1} \exp \left[3^{-d n} C \lambda^{\tau+1 / 2}|\Lambda|\right],
\end{aligned}
$$

where we have used the estimate $\left|R_{n}\right| \leqq \sum_{x \in \bar{\Lambda}_{n}}\left(2 b^{\prime} B^{2} \log \left(1+\left|p_{x}\right|\right)\right)^{d}$ and have assumed $\lambda$ to be small. This completes the proof of Proposition 4.3. 


\section{Effective Interactions}

In this chapter the graphical representation of the effective potential $V_{n}^{D}$ is derived, which allows us to prove Proposition 2.5. We recall here the basic formulae for $V_{n}^{D}$ :

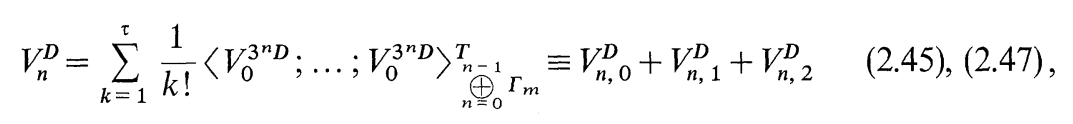

where

$$
V_{n, 0}^{D}=-\lambda M_{\mu \nu} \int_{D}: \nabla_{\mu} \psi_{x}^{n} \nabla_{v} \psi_{x}^{n}: d x-\lambda 3^{-n d} \int_{D}:\left(\nabla \psi_{x}^{n}\right)^{4}: d x
$$

and thus by (2.3) and (2.40)

$$
V_{0}^{3^{n} D}=V_{0,0}^{3^{n} D}-\sum_{k=2}^{\tau} \frac{1}{k !}\left\langle V_{0,0}^{3^{n} D} ; \ldots ; V_{0,0}^{3^{n} D}\right\rangle_{G_{0}}^{T} .
$$

We also defined $V_{n, 1}^{D}$ such that $\left\langle V_{n, 1}^{D}\right\rangle_{G_{n}}=0$. Thus by (2.45), (2.47), and (5.1)

$$
V_{n, 2}^{D}=\left\langle V_{n}^{D}\right\rangle_{G_{n}}=\sum_{k=1}^{\tau} \frac{1}{k !}\left[\left\langle\left\langle V_{0,0}^{3^{n} D} ; \ldots ; V_{0,0}^{3^{n} D}\right\rangle_{\oplus \Gamma_{m}}^{T}\right\rangle_{G_{n}}-\left\langle V_{0,0}^{3^{n} D} ; \ldots ; V_{0,0}^{3^{n} D}\right\rangle_{G_{0}}^{T}\right]
$$

and

$$
V_{n, 1}^{D}=\sum_{k=1}^{\tau} \frac{1}{k !}\left\langle V_{0,0}^{3^{3} D} ; \ldots ; V_{0,0}^{3^{n} D}\right\rangle_{\oplus \Gamma_{m}}^{T}-V_{n, 0}^{D}-\text { constant. }
$$

We can now easily write down the "Feynman rules" for $V_{n, 1}^{D}$ and $V_{n, 2}^{D}$ using (2.48), (5.2), and (5.3) together with the decomposition of $\nabla \psi^{0}$ given in (2.46).

Namely consider vertices $v_{\varrho}$, carrying indices $\mu_{Q^{2}}, \ldots \in\{1, \ldots, d\}$ and $z_{Q} \in \Lambda^{n}$ (which for brevity we suppress):

$$
v_{\varrho}={ }_{\lambda_{\varrho}}^{v_{\varrho}}>^{z_{\varrho}}<{ }_{\kappa_{\varrho}}^{\mu_{\varrho}}=\lambda \delta_{\mu_{\varrho} \kappa_{\varrho}} \delta_{v_{\varrho} \lambda_{\varrho}}
$$

or

$$
\mu_{\varrho} \stackrel{z_{\varrho}}{-} v_{\varrho}=\lambda M_{\mu_{Q} v_{\varrho}},
$$

where $M$ is defined in (2.41). Build connected graphs $\gamma$ by joining pairs of legs of $v_{e}$ 's, each element in the pair coming from a different vertex, to form lines of either of the following two types:

(1) hard lines

$$
H_{\mu v}\left(z, z^{\prime}\right)=\sum_{m=0}^{n-1} 3^{-d n}\left\langle\nabla_{\mu} \zeta_{3^{n-m_{z}}}^{m} \nabla_{v} \zeta_{3^{n-m_{z}}}^{m}\right\rangle_{\Gamma_{m}}
$$

coming from integrations with respect to $d \mu_{\oplus \Gamma_{m}}$,

(2) soft lines

$$
S_{\mu \nu}\left(z, z^{\prime}\right)=3^{-d n}\left\langle\nabla_{\mu} \psi_{z}^{n} \nabla_{\nu} \psi_{z^{\prime}}^{n}\right\rangle_{G_{n}}
$$

arising during Wick ordering with respect to $d \mu_{G_{n}}$ of the result of integration. 
Denote the subgraph composed of all vertices of $\gamma$ together with all hard (soft) lines by $H(\gamma)(S(\gamma))$, and the end points of a line $l$ by $z_{l_{-}}$and $z_{l_{+}}$. For a vertex $v_{\varrho}$ let $a_{\varrho}$ be the number of legs which are not contracted.

Given now such a graph $\gamma$, we assign to it the interaction $V_{n, \gamma}^{D}$ :

$$
\begin{aligned}
V_{n, \gamma}^{D}= & \frac{(-1)^{r(\gamma)}}{r(\gamma) !} 3^{d n r(\gamma)} \int_{D} \ldots \int_{D} \prod_{\varrho=1}^{r(\gamma)} v_{\varrho} \prod_{l \in H(\gamma)} H\left(z_{l_{-}}, z_{l_{+}}\right) \prod_{l \in S(\gamma)} S\left(z_{l_{-}}, z_{l_{+}}\right) \\
& \cdot: \prod_{\varrho: a_{\varrho}>0}\left(3^{-\frac{d n}{2}} \nabla \psi_{z_{\varrho}}^{n}\right)^{a_{\varrho}}:_{G_{n}} d z_{1} \ldots d z_{r(\gamma)} \\
\equiv & \int_{D} \ldots \int_{D} I_{\gamma}\left(z_{1}, \ldots, z_{r(\gamma)}\right): \prod_{a_{\varrho}>0}\left(\nabla \psi_{z_{\varrho}}^{n}\right)^{a_{\varrho}}:_{G_{n}} d z_{1} \ldots d z_{r(\gamma)},
\end{aligned}
$$

where for simplicity we have not written explicitly summations over indices of the gradients $\mu_{\varrho}, v_{\varrho}, \ldots$. following

$V_{n, 1}^{D}$ and $V_{n, 2}^{D}$ can be now described in terms of $V_{n, \gamma}^{D}$ as summarized in the

Lemma 5.1. Let $\mathscr{G}_{1}=\left\{\gamma: H(\gamma)\right.$ is connected and $\left.\sum a_{\varrho} \geqq 2\right\}$ and $\mathscr{G}_{2}=\{\gamma: H(\gamma)$ is not
connected, $\left.\sum a_{\varrho}=0\right\}$. Then connected, $\left.\sum_{\varrho} a_{\varrho}=0\right\}$. Then

$$
V_{n, i}^{D}=\sum_{\gamma \in \mathscr{G}_{i}} C(\gamma) V_{n, \gamma}^{D}, \quad i=1,2,
$$

where $C(\gamma)$ are combinatorical coefficients.

Proof. Because of truncation, the first term on the right hand side of (5.3) produces connected graphs of hard lines. Wick ordering does not produce new vertices, so $H(\gamma)$ 's will be connected. The resulting vacuum graphs will cancel the constant in (5.3) so $\sum a_{\varrho} \geqq 2$. This settles $i=1$. Similarly, in (5.2) the first term of the summand involves only $\gamma$ 's with $H(\gamma)$ connected which are cancelled by the second term since upon expanding $G_{0}=G_{n} \oplus\left(\bigoplus_{m=0}^{n-1} \Gamma_{m}\right)$ it involves all $\gamma$ 's. The factor $3^{\operatorname{dnr}(\gamma)}$ arises from the change of variables used in (2.46) $z \rightarrow 3^{n} z$.

Proposition 2.5 follows now from (5.6), (5.7) and the following claim:

Lemma 5.2. A) Let $\gamma \in \mathscr{G}_{2}$. Then for all $1 \leqq \varrho_{0} \leqq r(\gamma)$

$$
\int_{\Lambda^{n}} \ldots \int_{\Lambda^{n}}\left|I_{\gamma}\left(z_{1}, \ldots, z_{r(\gamma)}\right)\right| \prod_{\varrho \neq \varrho_{0}} d z_{\varrho} \leqq \lambda^{r(\gamma)} C\left(1+n^{C}\right)
$$

for $C$ independent of $n, \Lambda, \lambda$.

B) Let $\gamma \in \mathscr{G}_{1}$. Then (5.8) holds also with $I_{\gamma}$ replaced by

$$
I_{\gamma}\left(z_{1}, \ldots, z_{r(\gamma)}\right) \prod_{l \in H(\gamma)} \exp \varepsilon\left|z_{l_{-}}-z_{l_{+}}\right|_{p}
$$

for some $\varepsilon>0$. 
To prove Lemma 5.2 we shall need estimates for the falloff of the hard and soft lines:

$$
\begin{aligned}
\left|\left\langle\nabla_{\mu} \psi_{z}^{m} \nabla_{v} \psi_{z^{\prime}}^{m}\right\rangle_{G_{m}}\right| & \leqq C \frac{1}{1+\left|z-z^{\prime}\right|_{p}^{d}}, \\
\left|\left\langle\nabla_{\mu} \zeta_{z}^{m} V_{v} \zeta_{z^{\prime}}^{m}\right\rangle_{\Gamma_{m}}\right| & \leqq C e^{-\varepsilon\left|z-z^{\prime}\right|_{p}} .
\end{aligned}
$$

(5.9) and (5.10) are proved in Propositions A.7 and A.8. Proposition 5.2B follows now easily. We replace every soft line (5.5) by $3^{-d n} C$, every hard line (5.4) by

$$
C \sum_{m_{l}=0}^{n-1} 3^{-d m_{l}} \exp \left[-3^{n-m_{l}} \varepsilon\left|z_{l_{-}}-z_{l_{+}}\right|_{p}\right]
$$

and every vertex by $C \lambda$ obtaining

$$
\left|I_{\gamma}\right| \leqq C \lambda^{r(\gamma)} 3^{d n r(\gamma)-d n S-1 / 2 d n E} \sum_{\left(m_{l}\right)} \prod_{l \in H(\gamma)} 3^{-d m_{l}} e^{-\left.3^{n-m_{l \varepsilon}}\right|_{z_{-}-\left.z_{l_{+}}\right|_{p}}},
$$

where $S$ is the number of soft lines, $E$ that of external ones. By taking smaller $\varepsilon$ we can extract the extra convergence claimed in Proposition 5B. Since (recall $D \subset \Lambda^{n}$ )

$$
3^{-d m} \int_{\Lambda^{n}} d z e^{-3^{n-m_{\varepsilon}|z|_{p}}} \leqq C 3^{-d n}
$$

and $H(\gamma)$ is connected one easily gets from (5.11)

$$
\int_{\Lambda^{n}} \ldots \int_{\Lambda^{n}}\left|I\left(z_{1}, \ldots, z_{r(\gamma)}\right)\right| \prod_{\varrho \neq \varrho_{0}} d z_{\varrho} \leqq C \lambda^{r(\gamma)} 3^{d n-d n S-1 / 2 d n E} \sum_{\left(m_{l}\right)} 1 .
$$

But $E=\sum a_{\varrho} \geqq 2$ and $\sum_{\left(m_{l}\right)} 1=n^{C}$ which imply the claim.

Remark. Notice that for $\gamma$ with $E \geqq 4$ we get much better bound $C \lambda^{r}\left(1+n^{C}\right) 3^{-d n}$. The worst behaving terms are the marginal ones producing gaussian corrections to the measure.

In Proposition 5A we cannot use the exponential falloff which made 5B easy. However, $\gamma \in \mathscr{G}_{2}$ are vacuum graphs and thus one particle irreducible. We proceed as follows. First replace hard lines by

$$
\sum_{m_{l}=0}^{n-1} 3^{-m_{l} d} e^{-\varepsilon 3^{n-m_{l} \mid} \mid z_{l}-z_{l}+1},
$$

soft ones by (5.9) and vertices by $\lambda C$. Fix $m_{l}$ for each hard line and proceed with the following three steps:

1. Use the bounds

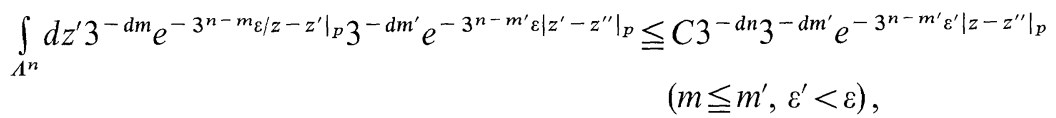

$$
\begin{aligned}
& \int_{A^{n}} d z^{\prime} 3^{-d m} e^{-3^{n-m_{\varepsilon} \mid z-z^{\prime}}} \frac{3^{-d n}}{1+\left|z^{\prime}-z^{\prime \prime}\right|^{-\varepsilon}} \leqq 3^{-d n} \frac{C 3^{-d n}}{1+\left|z-z^{\prime \prime}\right|^{d-\varepsilon}}, \\
& \int_{A^{n}} d z^{\prime} \frac{3^{-d n}}{1+\left|z-z^{\prime}\right|^{d-\varepsilon}} \cdot \frac{3^{-d n}}{1+\left|z^{\prime}-z^{\prime \prime}\right|^{d-\varepsilon}} \leqq 3^{-d n} \frac{C 3^{-d n}}{1+\left|z-z^{\prime \prime}\right|^{d-z^{\prime}}} \\
& \left(\varepsilon^{\prime}>\varepsilon\right)
\end{aligned}
$$

to get rid of two leg vertices $v_{\varrho}, \varrho \neq \varrho_{0}$, obtaining a new graph with only four leg vertices and lines decaying slightly slower. 
2. For four leg vertices without self contractions (these may arise in the first step) we use Schwartz inequality

$$
\int d z \prod_{\alpha=1}^{4}\left|L_{\alpha}\left(z-z_{\alpha}\right)\right| \leqq\left(\int d z \prod_{\alpha=1}^{2}\left|L_{\alpha}\left(z-z_{\alpha}\right)\right|^{2}\right)^{1 / 2}\left(\int d z \prod_{\alpha=3}^{4}\left|L_{\alpha}\left(z-z_{\alpha}\right)\right|^{2}\right)^{1 / 2}
$$

together with the estimates

$$
\begin{aligned}
& {\left[\int_{\Lambda^{n}} d z^{\prime}\left(3^{-d m} e^{\left.-3^{n-m_{\varepsilon} \mid z-z^{\prime}} \mid 3^{-d m^{\prime}} e^{-3^{n-m^{\prime}} \varepsilon \mid z^{\prime}-z^{\prime \prime}}\right)^{2}}\right]^{1 / 2}\right.}
\end{aligned}
$$

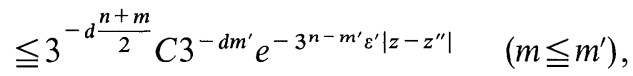

$$
\begin{aligned}
& {\left[\int_{\Lambda^{n}} d z^{\prime}\left(3^{-d m} e^{-3^{n-m_{\varepsilon}\left|z-z^{\prime}\right|}} \frac{3^{-d n}}{1+\left|z-z^{\prime}\right|^{d-\varepsilon}}\right)^{2}\right]^{1 / 2} \leqq 3^{-d \frac{n+m}{2}} C \frac{3^{-d n}}{1+\left|z-z^{\prime \prime}\right|^{d-\varepsilon}},} \\
& {\left[\int d z^{\prime}\left(\frac{3^{-d n}}{1+\left|z-z^{\prime}\right|^{d-\varepsilon}} \frac{3^{-d n}}{1+\left|z^{\prime}-z^{\prime \prime}\right|^{d-\varepsilon}}\right)^{2}\right]^{1 / 2} \leqq 3^{-d n} C \frac{3^{-d n}}{1+\left|z-z^{\prime \prime}\right|^{d-\varepsilon}} .}
\end{aligned}
$$

We can represent this pictorically by

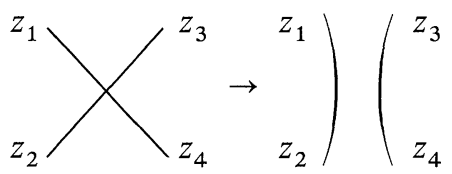

We pair $z_{\alpha}$ in (5.12) so, that the graph remains connected. This is always possible for a connected vacuum graph.

3. For four leg vertices with self contractions, i.e. $\supset$, the loop is easily bound, giving $C$ or $3^{-n d} C$ depending on whether it is hard or soft. The resulting two leg vertex is treated as in 1 .

After these operations we end up with $\infty$ or $\circ$ depending on whether $\varrho_{0}$ is a two or four leg vertex. Now apply 3 again and the result is the estimate

$$
\int_{\Lambda^{n}} \ldots \int_{\Lambda^{n}}\left|I\left(z_{1}, \ldots, z_{r(\gamma)}\right)\right| \prod_{\varrho \neq \varrho} d z_{\varrho} \leqq \lambda^{r(\gamma)} C 3^{d n-d n x}\left(1+\sum_{\left(m_{l}\right)} 1\right),
$$

where $x$ is the number of soft lines disposed of in step 3. We started with at least two soft lines and at least one of them enters step 3. Hence $x \geqq 1$ and (5.13) gives the claim since $\sum_{\left(m_{l}\right)} 1=n^{C}$.

\section{Probability Estimates}

We will prove in this chapter the probability estimates for the fluctuation integral stated in Lemmas 3.2 and 4.5. The proofs are simple adaptations of the superstability estimates of Ruelle [6]. Our situation is, however, slightly different from that of [6]; this chapter will hence be self-contained.

Note that the characteristic functions $\chi_{\bar{B}}$ in (3.8) are a special case of $\chi_{\bar{p}}$ in (4.10), namely $\chi_{\bar{B}}=\chi_{\bar{p}=0}$. We can now treat Lemmas 3.2 and 4.5 on the same footing by 
considering

where

$$
\begin{aligned}
\left\langle\left|\frac{\delta \log \chi_{p_{y}}\left(\xi_{y}^{n}\right)}{\delta \xi_{y}^{n}}\right|\right\rangle_{\bar{p}, t} & \equiv \bar{Z}^{-1} \sum_{m=p_{y} \pm 1} \int e^{\bar{U}^{E}} \delta\left(\xi_{y}^{n}-m \bar{B}\right) \prod_{x \neq y} \chi_{p_{x}}\left(\xi_{x}^{n}\right) \prod_{x} d \xi_{x}^{n} \\
& \equiv S^{+}\left(p_{y}, E\right)+S^{-}\left(p_{y}, E\right),
\end{aligned}
$$

$$
\bar{U}^{E}\left(\xi^{n}\right)=t V_{n}^{E}\left(3^{-d / 2} \nabla \psi_{1 / 3}^{n+1}+\nabla \zeta^{n}\right)-1 / 2\left(\xi^{n}, \Gamma_{n}^{-1} \xi^{n}\right)
$$

and in the case of Lemma $4.5 E=\tilde{\Lambda}^{n}=\Lambda^{n} \backslash\left(3 D_{n+1} \cup R_{n}\right)$ whereas for Lemma 3.2 $E=\Lambda^{n}$ and in both cases the $\nabla \psi^{n+1}$ occuring in (6.2) satisfies $\left\|\nabla \psi^{n+1}\right\|_{L^{\infty}(E)} \leqq B$. Now (3.20) and (4.21) of the lemmas follow from

Proposition 6.1. A) Let $E=\Lambda^{n}$ or $\tilde{\Lambda}^{n}$. Then for $b$ large, $\lambda$ small $S^{ \pm}(0, E) \leqq e^{-C \bar{B}^{2}}$.

B) Let $b$ and $b^{\prime}$ be large enough and $\lambda$ be small. Then

$$
\sup _{\bar{p}, t, y: p_{y} \neq 0} e^{-\varepsilon d\left(y, \tilde{\Lambda}_{n}\right)} S^{ \pm}\left(p_{y}, \tilde{\Lambda}^{n}\right) \leqq e^{-C \bar{B}^{2}} .
$$

We shall perform a translation in the integral (6.1) so that all the characteristic functions are centered at the origin i.e. $\xi \rightarrow \xi+\bar{B} \bar{p}$. Denoting (we suppress $n$ )

$$
U^{E}(\xi)=\bar{U}^{E}(\xi+\bar{B} \bar{p})-\bar{U}^{E}(\bar{B} \bar{p})-\left(\xi, u^{E}\right),
$$

where

we obtain

$$
u^{E}=\left.\frac{\delta \bar{U}^{E}}{\delta \xi}\right|_{\xi=\bar{B} \bar{p}}
$$

$$
S^{ \pm}\left(p_{y}, E\right)=e^{ \pm \bar{B} u_{y}^{E}} Z^{-1} \int e^{u^{E}(\xi)} \mu\left(d \xi_{\bar{\Lambda}_{n} \backslash y}\right) .
$$

In (6.5), to coincide with the notation of [6], we have defined

$$
\mu\left(d \xi_{A}\right)=\prod_{x \in A} \chi_{\bar{B}}\left(\xi_{x}\right) e^{\xi_{x} u u_{x}^{E}} d \xi_{x}
$$

and $\xi$ in $U^{E}(\xi)$ is restricted to be equal $\pm \bar{B}$ at $y$. Denote also by $\xi_{A}$ the configuration with $\xi_{x}$ put to zero for $x \in A^{c}$. We will need the following properties of $U^{E}$ :

Lemma 6.2. Let $U$ denote $U^{E}$ with $E$ either of the sets in Proposition 6.1 and let $\lambda$ be sufficiently small.

A) There exist constants $A_{1}, A_{2}>0$ such that

for all $B \subset \bar{\Lambda}_{n}$.

$$
-A_{2} \sum_{x \in B} \xi_{x}^{2} \leqq U\left(\xi_{B}\right) \leqq-A_{1} \sum_{x \in B} \xi_{x}^{2}
$$

B) Let $A, B \subset \bar{\Lambda}_{n}$ and define

$$
W\left(\xi_{A}, \xi_{B}\right)=U\left(\xi_{A \cup B}\right)-U\left(\xi_{A}\right)-U\left(\xi_{B}\right) .
$$

Then for some $C, \varepsilon>0$

$$
\left|W\left(\xi_{A}, \xi_{B}\right)\right| \leqq C \sum_{x \in A, y \in B} e^{-\varepsilon|x-y|_{p}}\left(\xi_{x}^{2}+\xi_{y}^{2}\right) .
$$

Remark. (6.7) and (6.9) are the standard conditions for superstability and regularity 
[6]. For the other cases however we must slightly modify the arguments in [6] due to the fact that for some $x u_{x}^{E}$ in the single spin measure (6.6) can be very large.

Proof. The change of variables $\xi \rightarrow \xi+\bar{B} \bar{p}$ induces

$$
\nabla \zeta_{z} \rightarrow \nabla \zeta_{z}+\nabla \zeta_{z}^{\prime} \equiv \nabla \zeta_{z}+\nabla(\mathscr{A} Q \bar{B} \bar{p})_{z}
$$

Now $V_{n}^{\tilde{\Lambda}^{n}}$ involves only $\zeta_{z}$ for $z \in R_{n}^{c}$ (in case of $V^{\Lambda^{n}}, \bar{p}=0$ and so $\zeta^{\prime}=0$ ). Thus for

$$
p_{y} \neq 0|z-y|_{p} \geqq b^{\prime} B^{2} \log \left(1+\left|p_{y}\right|\right)
$$

and as previously we get for $b^{\prime}$ large $\left\|\nabla \zeta^{\prime}\right\| \leqq 1$. Absorbing $\nabla \zeta^{\prime}$ to $\nabla \psi$ it thus suffices to prove the lemma for

$$
U^{E}(\xi) \equiv \bar{U}^{E}(\xi)-\bar{U}^{E}(0)-\left(\left.\frac{\delta \bar{U}^{E}}{\delta \xi}\right|_{\xi=0}, \xi\right)
$$

when $\|\nabla \psi\|_{L^{\infty}(E)} \leqq B+1$ and $\|\xi\|_{\infty} \leqq \bar{B}$. The "free" part of $U^{E},-1 / 2\left(\xi, \Gamma^{-1} \xi\right)$, satisfies A) and $\mathrm{B}$ ) since $A>\Gamma^{-1} \geqq A^{\prime}>0$ and has exponential falloff (see Proposition A.3). It is easy to infer from Proposition 2.5 that $U+1 / 2\left(\xi, \Gamma^{-1} \xi\right)$ is a sum of terms

$$
\lambda \sum_{x_{i} \in \bar{\Lambda}_{n}} J\left(x_{1}, \ldots, x_{s}\right) \prod_{i=1}^{s} \xi_{x_{i}},
$$

where for $n \leqq \lambda^{-\delta}$ with $\delta$ small enough $\left|J e^{\varepsilon d\left(x_{1}, \ldots, x_{s}\right)}\right| \leqq \lambda C B^{C}$. Since $\left|\xi_{x}\right| \leqq \bar{B}$ and $s \geqq 2$ because of the subtraction in (6.10), we can bound (6.11) by

$$
\lambda C B^{C} \sum_{x, y \in \bar{\Lambda}_{n}} e^{-\varepsilon|x-y|} \xi_{x} \xi_{y} .
$$

Thus (6.10) is regular and for $\lambda$ small enough does not change the superstability when added to $-1 / 2\left(\xi, \Gamma^{-1} \xi\right)$.

Let us now prove Proposition 6.1B: From (6.5) we get using (6.6) and (6.8)

$$
\begin{aligned}
& S^{ \pm}\left(p_{y}, \tilde{\Lambda}^{n}\right)=e^{ \pm \bar{B} u_{\hat{y}}^{\tilde{x}^{n}}} e^{U\left(\xi_{y}\right)} \uparrow_{\xi_{y}= \pm \bar{B}}\left(\int_{-\bar{B}}^{\bar{B}} d \xi e^{\xi \mu_{\bar{y}}^{\tilde{x}^{n}}+U(\xi)}\right)^{-1} Z^{-1} \\
& \cdot \int \mu\left(d \xi_{y}^{\prime}\right) e^{U\left(\xi_{y}^{\prime}\right)} \int \mu\left(d \xi_{\bar{A}_{n} \mid y}\right) e^{W\left(\xi_{y}^{\prime}, \xi_{\bar{A}_{n}} \backslash y\right)+U\left(\xi_{\bar{A}_{n} \backslash y}\right)} \\
& \cdot e^{W\left(\xi_{y}, \xi_{\bar{A}_{n} \backslash y}\right)-W\left(\xi_{y}^{\prime}, \xi_{\bar{A}_{n} \backslash y}\right)} \uparrow_{\xi_{y}= \pm \bar{B}}
\end{aligned}
$$

Since $\|\xi\|_{\infty} \leqq \bar{B}$, we can use Lemma $6.2 \mathrm{~A}$ and $\mathrm{B}$ to estimate

$$
e^{U\left(\zeta_{y}\right)+W\left(\zeta_{y}, \zeta_{\bar{A}_{n}} \backslash y\right)-W\left(\xi_{y}^{\prime}, \bar{\zeta}_{\bar{A}_{n} \backslash y}\right)} \leqq e^{C \bar{B}^{2}} .
$$

Thus by (6.13), (6.7), and (6.14)

$$
S^{ \pm}\left(p_{y}, \tilde{\Lambda}^{n}\right) \leqq e^{C \bar{B}^{2}} e^{ \pm \bar{B} u \hat{\Lambda}_{y}^{n}}\left[\int d \xi e^{\xi u \tilde{y}_{y}^{n}}\right]^{-1} \leqq\left[1+\left|u_{y}^{\tilde{\Lambda}^{n}}\right|\right] e^{C \bar{B}^{2}}
$$

We write $u_{y}^{\tilde{A}^{n}}$ using (6.4) and (6.2) as

$$
u_{y}^{\tilde{\Lambda}^{n}}=-\bar{B}\left(\Gamma^{-1} \bar{p}\right)_{y}+\left.t \frac{\delta V_{n}^{\tilde{\Lambda}^{n}}}{\delta \xi_{y}}\right|_{\xi=\bar{B} \bar{p}} .
$$

As in the proof of Lemma $6.2\left\|\nabla \zeta \uparrow_{\xi=\bar{B} \bar{p}}\right\|$ is small since $z \in R_{n}^{c}$ and the second term in (6.16) can be bounded by one (say). Hence, to obtain the claim we have to prove

$$
\sup _{y: p_{y} \neq 0} e^{-\varepsilon d\left(y, \tilde{A}_{n}\right)}\left[1+\left|\bar{B}\left(\Gamma^{-1} \bar{p}\right)_{y}\right|\right] e^{C \bar{B}^{2}} \leqq e^{-C \bar{B}^{2}} .
$$


Using the exponential falloff of $\Gamma^{-1}$ we get

$$
\begin{aligned}
& e^{-\varepsilon d\left(y, \tilde{A}_{n}\right)}\left[1+\left|\bar{B}\left(\Gamma^{-1} \bar{p}\right)_{y}\right|\right] \\
& \quad \leqq e^{-\varepsilon b^{\prime} B^{2} \log \left(1+\left|p_{y}\right|\right)}\left(1+\bar{B} \sum_{x: p_{x} \neq 0} e^{-\varepsilon d\left(x, \tilde{A}^{n}\right)}\left|p_{x}\right| e^{-\varepsilon|x-y|_{p}}\right) \\
& \quad \leqq C \bar{B} e^{-b^{\prime} B^{2}}
\end{aligned}
$$

choosing $b^{\prime}$ large enough (6.17) follows from (6.18).

Proof of Proposition 6.1 A. Following [6] we insert a partition of unity in the integral of (6.5). For this purpose, let $0<\psi_{0}<\psi_{1}<\ldots<\psi_{p}$ and let $V_{0} \subset V_{1} \subset \ldots \subset V_{p}=\bar{\Lambda}_{n}$ be cubes in $\bar{A}_{n}$ centered at $y$ such that $\psi_{0}\left|V_{0}\right|=\bar{B}^{2}, \psi_{p-1} \leqq \bar{B}^{2}$ and $\psi_{p}>\bar{B}^{2}$. Consider the sets

$$
R_{q}=\left\{\xi: \sum_{x \in V_{q}} \xi_{x}^{2} \geqq \psi_{q}\left|V_{q}\right|, \sum_{x \in V_{q+k}} \xi_{x}^{2}<\psi_{q+k}\left|V_{q+k}\right|, k>0\right\} .
$$

$\left\{R_{q}\right\}_{q \leqq p-1}$ forms a partition of unity since $\left|\xi_{y}\right|=\bar{B}$ and $\|\xi\|_{\infty} \leqq \bar{B}$. Thus (we denote $V_{q}$ by $q$ for brevity)

$$
\begin{aligned}
& Z^{-1} \int e^{U\left(\check{\check{\epsilon}} \bar{A}_{n}\right)} \mu\left(d \xi_{\bar{A}_{n} \backslash y}\right) \\
& =\sum_{q=1}^{p-1} Z^{-1} \int_{R_{q-1}} e^{U\left(\xi_{q}\right)+U\left(\xi_{p \backslash q}\right)+W\left(\xi_{q}^{\prime}, \xi_{p} \backslash q\right)} e^{W\left(\xi_{q}, \xi_{p \backslash q}\right)-W\left(\xi_{q}^{\prime}, \xi_{p \backslash q}\right)} \mu\left(d \xi_{p \backslash y}\right) \\
& +Z^{-1} \int_{R_{p-1}} e^{U\left(\xi_{p-1}\right)+U\left(\xi_{p} \backslash_{p-1}\right)+W\left(\xi_{p-1}^{\prime}, \xi_{p} \backslash_{p}-1\right)} e^{W\left(\xi_{p-1}, \xi_{p} \backslash_{p-1}\right)-W\left(\xi_{p-1}^{\prime}, \xi_{p} \backslash_{p-1}\right)} \mu\left(d \xi_{p \backslash y}\right) \\
& \leqq \sum_{q=1}^{p-1} \varepsilon(q) Z^{-1} \int_{\Sigma_{q}} e^{U\left(\xi_{q}^{\prime}\right)} \mu\left(d \xi_{q}^{\prime}\right) \int e^{U\left(\xi_{p \backslash q}\right)+W\left(\xi_{q}^{\prime}, \xi_{p \backslash q}\right)} \mu\left(d \xi_{p \backslash q}\right) \\
& +\varepsilon^{\prime}(p) Z^{-1} \int_{\Sigma_{p-1}} e^{U\left(\xi_{p-1}^{\prime}\right)} \mu\left(d \xi_{p-1}^{\prime}\right) \int e^{U\left(\xi_{p} \backslash_{p-1}\right)+W\left(\xi_{p-1}^{\prime}, \xi_{p \backslash p-1}\right)} \mu\left(d \xi_{p \backslash p-1}\right),
\end{aligned}
$$

where $\Sigma_{q}$ is $\left\{\xi_{q}:\left|\xi_{x}\right| \leqq 1\right\}$ and

$$
\begin{aligned}
& \varepsilon(q)=\int_{\left\|\xi_{q-1}\right\|_{2}^{2} \geqq \psi_{q-1} V_{q-1}} e^{-A_{1}\left\|\xi_{q}\right\|_{2}^{2}} \mu\left(d \xi_{q}\right)\left(\int_{\Sigma_{q}} e^{U\left(\xi_{q}\right)} \mu\left(d \xi_{q}\right)\right)^{-1} \sup _{\xi \in A_{q} \cup R_{q}} \mathrm{e}^{2\left|W\left(\xi_{q}, \xi_{p} \backslash q\right)\right|} \\
& \varepsilon^{\prime}(p)=\int_{\left\|\xi_{p-1}\right\|_{2}^{2} \geqq \psi_{p-1} V_{p-1}} e^{-A_{1}\left\|\xi_{p-1}\right\|_{2}^{2}} \mu\left(d \xi_{p-1}\right)\left(\int_{\Sigma_{p-1}} e^{U\left(\xi_{p-1}\right)} \mu\left(d \xi_{p-1}\right)\right)^{-1} \\
& \sup _{\xi \in A_{p-1} \cup R_{p-1}} e^{2 \mid W\left(\xi_{p-1}, \xi_{p} \backslash_{p-1}\right)} .
\end{aligned}
$$

We denoted by $A_{q}$ the set $\left\{\xi:\left\|\xi_{q}\right\|_{\infty} \leqq 1\right.$ and $\left.\left\|\xi_{q+k}\right\|_{2}^{2}<\psi_{q+k} V_{q+k}\right\}$. It will be shown below that for $b$ sufficiently large we can choose $\left\{\psi_{q}, V_{q}\right\}$ such that

$$
\begin{aligned}
\varepsilon(q) & \leqq 2^{-q} e^{-C \bar{B}^{2}}, \\
\varepsilon^{\prime}(p) & \leqq 2^{-p} e^{-C \bar{B}^{2}},
\end{aligned}
$$

and thus from (6.5) and (6.20)

$$
S^{ \pm}(0, E) \leqq e^{ \pm \bar{B} u \frac{E}{y}} e^{-C \bar{B}^{2}}
$$

Let first $E=\tilde{\Lambda}^{n}$. As in the previous proof we only need to consider the contribution 
and by the exponential falloff of $\Gamma^{-1}$ for $b^{\prime}$ large $\left|\bar{B}\left(\Gamma^{-1} \bar{p}\right)_{y}\right| \leqq 1$. So

$$
S^{ \pm}\left(0, \tilde{\Lambda}^{n}\right) \leqq e^{-C \bar{B}^{2}}
$$

which is the claim. In case of $E=\Lambda^{n}, \bar{p}=0$ and only $V^{\Lambda^{n}}$ in (6.2) contributes to $U_{y}^{E}$. Again, for $\lambda$ small $\left|u_{y}^{E}\right| \leqq 1$ and

$$
S^{ \pm}\left(0, \Lambda^{n}\right) \leqq e^{-C \bar{B}^{2}}
$$

completing the proof. Finally let us prove (6.23) and (6.24). Note that by (6.6) and (6.7)

$$
\int_{\Sigma_{q}} e^{U\left(\xi_{q}\right)} \mu\left(d \xi_{q}\right) \geqq C^{\left|V_{q}\right|} \prod_{x \in q} \int_{-1}^{1} e^{\xi_{u} u_{x}^{E}} d \xi \geqq C^{\left|V_{q}\right|}
$$

since $\int_{-1}^{1} e^{a \xi} d \xi>1$. Hence from (6.21) and (6.22)

$$
\begin{aligned}
& \varepsilon(q) \leqq C^{\left|V_{q}\right|} e^{-C \psi_{q-1} V_{q-1}} \sup _{\xi \in A_{q} \cup R_{q}} e^{2\left|W\left(\xi_{q}, \xi_{p \backslash q}\right)\right|} \int \mu\left(d \xi_{q}\right) \\
& \varepsilon^{\prime}(p) \leqq C^{\left|V_{p-1}\right|} e^{-C \psi_{p-1} V_{p-1}} \sup _{\xi \in A_{p-1} \cup R_{p-1}} e^{2\left|W\left(\xi_{p-1}, \xi_{p} \backslash_{p-1}\right)\right|} \int \mu\left(d \xi_{p-1}\right) .
\end{aligned}
$$

By (6.9) and $\|\xi\|_{\infty} \leqq \bar{B}$ we also get

$$
\left|W\left(\xi_{p-1}, \xi_{p \backslash p-1}\right)\right| \leqq C \bar{B}^{2}\left|\partial V_{p-1}\right|
$$

and

$$
\begin{aligned}
\left|W\left(\xi_{q}, \xi_{p \backslash q}\right)\right| & \leqq C \sum_{\substack{x \in q \\
y \in p \backslash q}} e^{-\varepsilon|x-y|}\left(\xi_{x}^{2}+\xi_{y}^{2}\right) \\
& =C\left[\sum_{\substack{x \in q-1 \\
y \in p \backslash q}} e^{-\varepsilon|x-y|} \xi_{x}^{2}+\sum_{\substack{x \in q \backslash q-1 \\
y \in p \backslash q}} e^{-\varepsilon|x-y|} \xi_{x}^{2}+\sum_{\substack{x \in q \\
y \in p \backslash q}} e^{-\varepsilon|x-y|} \xi_{y}^{2}\right] \\
& \equiv W_{1}+W_{2}+W_{3} .
\end{aligned}
$$

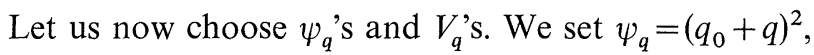

$$
V_{q}=y+\left[-r_{0}-q r_{1}, r_{0}+q r_{1}\right]^{d}
$$

and $p$ as the largest integer such that $q_{0}+p-1 \leqq \bar{B}$. Also, $q_{0}=\bar{B}^{-1 / 2}$ and $r_{0}=1 / 2(\bar{B})^{1 / d}$ and thus $\psi_{0}\left|V_{0}\right|=\bar{B}^{2}$ as required. $r_{1}$ will be chosen in a $\bar{B}$ independent way below. Let $\xi \in R_{q-1}$ (the other case $\xi \in A_{q-1}$ is similar). Noting that

$$
\operatorname{dist}(q, q+k \backslash q+k-1)=r_{1}(k-1)
$$

for $k \geqq 1$ we estimate the $W_{i}$ in (6.29):

$$
\begin{aligned}
W_{1} \leqq & C \psi_{q}\left|V_{q}\right| e^{-\varepsilon r_{1}}, \\
W_{2} \leqq & C\left(\psi_{q}\left|V_{q}\right|-\psi_{q-1}\left|V_{q-1}\right|\right), \\
W_{3} \leqq & C \sum_{k=1}^{p-q-1} \sum_{\substack{y \in q+k \backslash q+k-1\\
}} e^{-\varepsilon r_{1}(k-1) \xi_{y}^{2}} \\
& +C\left|\partial V_{q}\right| \bar{B}^{2} e^{-m r_{1}(p-q-1)} .
\end{aligned}
$$


Denoting $\sum_{x \in q+k} \xi_{x}^{2}$ by $E_{q+k}$ we get $(q \leqq p-2)$

$$
\begin{aligned}
& \sum_{k=1}^{p-q-1} \sum_{y \in q+k \backslash q+k-1} e^{-\varepsilon r_{1}(k-1)} \xi_{y}^{2}=\sum_{k=1}^{p-q-1} e^{-\varepsilon r_{1}(k-1)}\left(E_{q+k}-E_{q+k-1}\right) \\
& \leqq E_{q+1}-E_{q}+\sum_{k=1}^{p-q-2} e^{-\varepsilon r_{1} k} E_{q+k+1} \leqq \psi_{q-1}\left|V_{q-1}\right| \sum_{k=1}^{p-q-2} e^{-\varepsilon r_{1} k} \frac{\psi_{q+k+1}\left|V_{q+k+1}\right|}{\psi_{q-1}\left|V_{q-1}\right|} \\
& \quad+\left(\psi_{q+1}\left|V_{q+1}\right|-\psi_{q-1}\left|V_{q-1}\right|\right)+\left(\psi_{q}\left|V_{q}\right|-\psi_{q-1}\left|V_{q-1}\right|\right)
\end{aligned}
$$

From the definitions of $\psi_{q}$ and $V_{q}$ we obtain

$$
\begin{gathered}
\frac{\psi_{q+k+1}\left|V_{q+k+1}\right|}{\psi_{q-1}\left|V_{q-1}\right|}=\left(1+\frac{k+2}{q_{0}+q-1}\right)^{2}\left(1+\frac{(k+2) r_{1}}{r_{0}+(q-1) r_{1}}\right)^{d} \\
\psi_{q+i}\left|V_{q+i}\right|-\psi_{q-1}\left|V_{q-1}\right|=\psi_{q-1}\left|V_{q-1}\right|\left[\left(1+\frac{1+i}{q_{0}+q-1}\right)^{2}\left(1+\frac{(1+i) r_{i}}{r_{0}+(q-1) r_{1}}\right)^{d}-1\right] .
\end{gathered}
$$

Inserting (6.34) and (6.35) to (6.30)-(6.33) we deduce for $q \leqq p-2$ :

$$
\begin{aligned}
\left|W\left(\xi_{q}, \xi_{p \backslash q}\right)\right| \leqq & C\left[e^{-\varepsilon r_{1}}+\frac{1}{q_{0}}+\frac{r_{1}}{r_{0}}\right] \psi_{q-1}\left|V_{q-1}\right| \\
& +C\left(r_{0}+q r_{1}\right)^{d-1} \bar{B}^{2} e^{-\varepsilon r_{1}(p-q-1)} .
\end{aligned}
$$

The second term in (6.36) is bounded easily by (recall that $q_{0}+p \geqq \bar{B}$ )

$$
C \psi_{q-1}\left|V_{q-1}\right| \frac{1}{r_{0}+q r_{1}} \frac{\left(p+q_{0}-2\right)^{2}}{\left(q_{0}+q-1\right)^{2}} e^{-\varepsilon r_{1}(p-q-1)} \leqq C \bar{B}^{-1 / 2} \psi_{q-1}\left|V_{q-1}\right| \text {. }
$$

Recalling that $q_{0}=\bar{B}^{1 / 2}$ and $r_{0}=1 / 2(\bar{B})^{1 / d}(6.36)$ and (6.37) inserted to (6.26) give for $r_{1}$ large enough

$$
\varepsilon(q) \leqq e^{-C \psi_{q-1}\left|V_{q}-1\right|} \int \mu\left(d \xi_{q}\right)
$$

Similarly, from (6.28)

$$
\left|W\left(\xi_{p-1}, \xi_{p \backslash p-1}\right)\right| \leqq C \bar{B}^{2} p^{-1}\left|V_{p-1}\right| \leqq C \bar{B}^{-1} \psi_{p-1}\left|V_{p-1}\right|
$$

and thus

$$
\varepsilon^{\prime}(p) \leqq e^{-C \psi_{p-1}\left|V_{p-1}\right|} \int \mu\left(d \xi_{p-1}\right)
$$

Now

$$
\int \mu\left(d \xi_{q-1}\right) \leqq C \bar{B}^{\left|V_{q-1}\right|} \sup _{x \in q-1} \exp \left[\left|\bar{B} u_{x}^{\tilde{\Lambda}_{n}}\right|\left|V_{q-1}\right|\right] .
$$

Now recall that by the choice of $p, \max _{x \in p-1}|x-y| \leqq C \bar{B}$. On the other hand, for any $u$, $|y-u| \geqq b^{\prime} B^{2} \log \left(1+\left|p_{u}\right|\right)$. Thus as before, for $b^{\prime}$ large enough $\left|u_{x}^{\tilde{A}^{n}}\right|$ is small for all $x \in p-1$ and

$$
\int \mu\left(d \xi_{q-1}\right) \leqq C e^{C \bar{B}\left|V_{q-1}\right|} q \leqq p .
$$

(6.38)-(6.40) imply now (6.23) and (6.24). 
In above we have assumed, that the boxes $V_{0}, \ldots, V_{p-1}$ are entirely inside $\bar{\Lambda}_{n}$. The case when it does not happen is even simpler and necessary modifications are left to the reader.

Finally, we wish to prove (3.21) and (4.22) of the lemmas. To this end notice that

$$
\begin{aligned}
\int \chi_{\bar{B}}\left(\xi^{n}\right) d \mu_{\Gamma_{n}} & =\exp \left[-\int_{\bar{B}}^{\infty} d \beta \frac{d}{d \beta} \ln \int \chi\left(\left|\xi^{n}\right| \leqq \beta\right) d \mu_{\Gamma_{n}}\right] \\
& =\exp \left[-\int_{\bar{B}}^{\infty} d \beta \sum_{x \in \bar{A}_{n}} \frac{2 \int \delta\left(\xi_{x}^{n}-\beta\right) \prod_{y \neq x} \chi\left(\left|\xi_{y}^{n}\right| \leqq \beta\right) d \mu_{\Gamma_{n}}}{\int \prod_{y} \chi\left(\left|\xi_{y}^{n}\right| \leqq \beta\right) d \mu_{\Gamma_{n}}}\right] \\
& =\exp \left[-\int_{\bar{B}}^{\infty} d \beta \sum_{x \in \bar{\Lambda}_{n}} 2 Z^{-1} \int \delta\left(\xi_{x}^{n}-\beta\right) e^{U_{0}} \prod_{y \neq x} \chi\left(\left|\xi_{y}^{n}\right| \leqq \beta\right) \prod_{y} d \xi_{y}^{n}\right]
\end{aligned}
$$

where $U_{0}$ is superstable and regular. Similarly,

$$
\begin{gathered}
\int \prod_{y: p_{y} \neq 0} \chi_{p_{y}}\left(\xi_{y}^{n}\right) d \mu_{\Gamma_{n}}=\int \prod_{y: p_{y} \neq 0} \chi_{p_{y}}\left(\beta_{y}\right) d \beta_{y} \int \prod_{y: p_{y} \neq 0} \delta\left(\xi_{y}^{n}-\beta_{y}\right) d \mu_{\Gamma_{n}} \\
\quad=\int \prod_{y: p_{y} \neq 0} \chi_{p_{y}}\left(\beta_{y}\right) d \beta_{y} \cdot Z^{-1} \int \prod_{y: p_{y} \neq 0} \delta\left(\xi_{y}^{n}-\beta_{y}\right) e^{U_{0}} \prod_{y} d \xi_{y}^{n} .
\end{gathered}
$$

By [6] we can find $C>0$ and $\delta$ such that

$$
Z^{-1} \int \prod_{y \in Y} \delta\left(\xi_{y}^{n}-\beta_{y}\right) e^{U_{0}} \prod d \xi_{y}^{n} \leqq \exp \left[\sum_{y \in Y}\left(-C \beta_{y}^{2}+\delta\right)\right]
$$

and the same holds if the domain of integration over $\xi_{y}^{n}$ 's is restricted to an interval. Now (3.21) and (4.22) follow easily from (6.41) and (6.42).

\section{Appendix}

We shall prove in this Appendix the various properties of the covariances $G_{m}$ and $\Gamma_{m}$ and the operators $\mathscr{A}_{m}$ and $\nabla \mathscr{A}_{m}$ needed in the text.

Let us start with $G_{m}$. A straightforward computation using (2.1), (1.7), and (2.26) yields

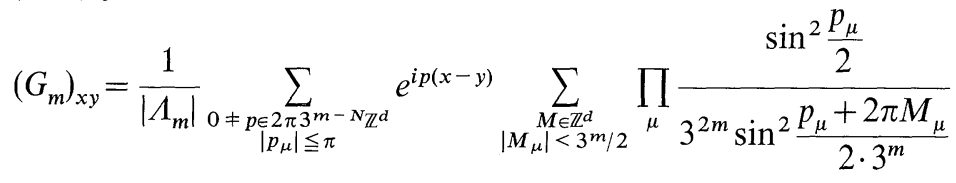

$$
\begin{aligned}
& \cdot\left[3^{2 m} \mu\left(\frac{p+2 \pi M}{3^{m}}\right)\right]^{-1} \equiv \frac{1}{|\Lambda|} \sum_{0 \neq p} \hat{G}_{m}(p) e^{i p(x-y)},
\end{aligned}
$$

where we recall that $\mu(p)=2 \sum_{\mu=0}^{d-1}\left(1-\cos p_{\mu}\right)[($ A. 1$)$ is easiest to derive by first computing it in infinite volume and then periodizing]. We will consider $\hat{G}_{m}(p)$ as defined on the periodic box $[-\pi, \pi]^{d}$. Notice that it is non-vanishing and finite except for $p=0$. This shows invertibility of $G_{m}$ on the subspace $(I-E) \mathbb{R}^{\Lambda_{m}}$ (i.e. on 
sequences with vanishing means) and allows us to define $G_{m}^{-1}$ as the inverse of $G_{m}$ on this subspace:

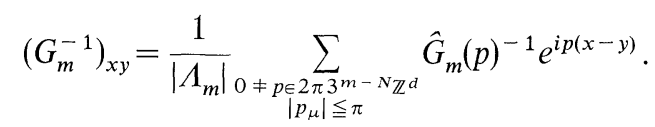

We gather in the following Lemma some properties of $\hat{G}_{m}$ needed later.

Lemma A.1. The functions $\hat{G}_{m}$ have the following properties:

a) $\quad \hat{G}_{m+1}(p)=\sum_{q: q_{\mu}=0, \pm 1} 3^{-2} \hat{G}_{m}\left(\frac{p+2 \pi q}{3}\right) \prod_{\mu}\left(\frac{1+2 \cos \frac{p_{\mu}+2 \pi q_{\mu}}{3}}{3}\right)^{2}$.

b) $\hat{G}_{m}$ has a unique continuation to a meromorphic function of complex $p_{0}$ for any $\mathbf{p} \equiv\left(p_{1}, \ldots, p_{d-1}\right) \in[-\pi, \pi]^{d-1}$ with two simple poles at the $p_{0}$ 's satisfying

$$
\operatorname{Re} p_{0}=0, \quad \operatorname{Im} p_{0}=3^{m} \operatorname{sh}^{-1}\left( \pm \sqrt{\sum_{\mu \neq 0} \sin ^{2} \frac{p_{\mu}}{2 \cdot 3^{m}}}\right) .
$$

c) There exists an $\varepsilon>0$, independent on $m$, such that for $\left|\operatorname{Im} p_{0}\right| \leqq \varepsilon$ and all $\mathbf{p} \in[-\pi, \pi]^{d-1}$

$$
C_{1}\left|3^{2 m} \mu\left(3^{-m} p\right)\right|^{-1} \leqq\left|\hat{G}_{m}(p)\right| \leqq C_{2}\left|3^{2 m} \mu\left(3^{-m} p\right)\right|^{-1}
$$

where $C_{1}>0$.

Proof. (a) follows from translating the infinite volume version of the first equality of (2.26) to momentum space. (b) follows by inspection from (A.1), the zeros of $\mu$ providing the poles. (c). Consider the function

$$
f(p)=\mu(p) \hat{G}_{m}(p)=\sum_{\substack{M \in \mathbb{Z}^{d} \\\left|M_{\mu}\right| \leqq 3^{m / 2}}} \prod_{\mu} \frac{\sin ^{2} 1 / 2 p_{\mu}}{3^{2 m} \sin ^{2} 1 / 23^{-m}\left(p_{\mu}+2 \pi M_{\mu}\right)} \frac{\mu(p)}{3^{2 m} \mu\left(\frac{p+2 \pi M}{3^{m}}\right)} .
$$

$f(p)$ is analytic in $p_{0}$ for $\left(\operatorname{Re} p_{0}, \mathbf{p}\right) \in[-\pi, \pi]^{d}$.

Let now $\left|\operatorname{Im} p_{0}\right|<\operatorname{ch}^{-1}(1 / 2)$. Then for $M \neq 0,\left|M_{\mu}\right| \leqq \frac{3^{m}}{2}$

$$
\begin{aligned}
\left|3^{2 m} \mu\left(\frac{p+2 \pi M}{3^{m}}\right)\right| & \geqq 23^{2 m}\left(\sum_{\mu>0}\left(1-\cos \frac{p_{\mu}+2 \pi M_{\mu}}{3^{m}}\right)+1\right. \\
& \left.-\cos \frac{\operatorname{Rep}_{0}+2 \pi M_{0}}{3^{m}} \operatorname{ch~Im} \frac{1}{3^{m}} p_{0}\right) \geqq C\left(1+M^{2}\right)
\end{aligned}
$$

and $\left|\frac{\sin \frac{p_{\mu}}{2}}{3^{m} \sin \frac{p_{\mu}+2 \pi M_{\mu}}{2 \cdot 3^{m}}}\right|^{2}=\frac{\sin ^{2} \frac{\operatorname{Rep}_{\mu}}{2}+\operatorname{sh}^{2} \frac{\operatorname{Imp}_{\mu}}{2}}{3^{2 m}\left(\sin \frac{\operatorname{Rep}_{\mu}+2 \pi M_{\mu}}{2 \cdot 3^{m}}+\operatorname{sh}^{2} \frac{\operatorname{Imp}_{\mu}}{2 \cdot 3^{m}}\right)} \leqq \frac{C}{1+M_{\mu}^{2}}$. 
Thus,

$$
|f(p)| \leqq C \text { for }\left|\operatorname{Im} p_{0}\right|<\operatorname{ch}^{-1} 1 / 2
$$

and hence by Cauchy's formula

$$
\left|\frac{d f}{d p_{0}}\right| \leqq C^{\prime} \text { for } \quad\left|\operatorname{Im} p_{0}\right| \leqq 1 / 2 \mathrm{ch}^{-1} 1 / 2 .
$$

Since $f(p)$ is strictly positive for $p$ real (A.6) implies that $|f(p)|>C_{1}>0$ for $\left|\operatorname{Im} p_{0}\right|$ small enough.

We can now turn to study the decay properties of the various kernels. We start with

Proposition A.2. There exist $C$ and $\varepsilon$ such that for all $m$ and $\Lambda$

$$
\left|\left(G_{m}^{-1}\right)_{x y}\right| \leqq C e^{-\varepsilon|x-y|_{p}} .
$$

Proof. By Lemma A.1(b) and (c) $\hat{G}_{m}(p)^{-1}$ is analytic in $p_{0}$ for $\left|\operatorname{Im} p_{0}\right|<\varepsilon$ and bounded uniformly in $m, \operatorname{Re} p \in[-\pi, \pi]^{d}$ and $\Lambda$. Thus

$$
\int_{\left|p_{\mu}\right| \leqq \pi} e^{i p(x-y)} \hat{G}_{m}(p)^{-1} d p=\int_{\left|p_{\mu}\right| \leqq \pi} e^{i p(x-y)} e^{-\frac{\varepsilon}{2}\left|x_{0}-y_{0}\right|_{p}} \hat{G}_{m}\left(p \pm i \frac{\varepsilon}{2} e_{0}\right)^{-1} d p
$$

which establishes exponential falloff of this kernel in zero direction, by symmetry in all coordinate directions and by $|x-y|_{p} \leqq C \max _{\mu}\left|x_{\mu}-y_{\mu}\right|_{p}$ in all directions. But

$$
\left(G_{m}^{-1}\right)_{x y}=\sum_{L \in 3^{N-m} \mathbb{Z}^{d}}(2 \pi)^{-d} \int_{\left|p_{\mu}\right| \leqq \pi} e^{i p(x-y-L)} \hat{G}_{m}(p)^{-1} d p
$$

and (A.7) follows.

Let us next consider the fluctuation covariances $\Gamma_{m}$. Their main properties are gathered in

Proposition A.3. A) $\Gamma_{m}^{-1}$ can be written as

$$
\Gamma_{m}^{-1}=Q^{T} G_{m}^{-1} Q
$$

where $Q$ is given by (2.14).

B) $\Gamma_{m}^{-1}$ and $\Gamma_{m}$ are strictly positive and satisfy

$$
\left.\begin{array}{l}
\left|\left(\Gamma_{m}^{-1}\right)_{x y}\right| \\
\left|\left(\Gamma_{m}\right)_{x y}\right|
\end{array}\right\} \leqq C e^{-\varepsilon|x-y|} .
$$

Proof. First note that $R^{T}: \mathbb{R}^{\bar{A}_{m}} \rightarrow \mathbb{R}^{\Lambda_{m}}$ and $Q^{T}: \mathbb{R}^{\Lambda_{m}} \rightarrow \mathbb{R}^{\bar{\Lambda}_{m}}$ are given by

$$
\begin{aligned}
& \left(R^{T} \xi\right)_{x}=\left\{\begin{array}{lll}
\xi_{x} & \text { if } & x \in \bar{\Lambda}_{m} \\
0 & \text { if } & x \in \Lambda_{m} \backslash \bar{\Lambda}_{m}
\end{array}\right. \\
& \left(Q^{T} \phi\right)_{x}=\phi_{x}-\phi_{y} .
\end{aligned}
$$

$y$ being the point in $\Lambda_{m} \backslash \bar{\Lambda}_{m}$ nearest to $x \in \bar{\Lambda}_{m}$. Thus by the definition of $\Gamma_{m}(2.28)$

$$
Q^{T} G_{m}^{-1} Q \Gamma_{m}=Q^{T} G_{m}^{-1} Q R\left(G_{m}-G_{m} C^{T} G_{m+1}^{-1} C G_{m}\right) R^{T} .
$$


As in the argument following (2.17) we can dispose of $Q R$ in (A.11) thus producing together with (A.10)

$$
Q^{T} G_{m}^{-1} Q \Gamma_{m}=Q^{T}(I-E) R^{T}-Q^{T}(I-E) C^{T} G_{m+1}^{T} C G_{m} R^{T} .
$$

Now, it is obvious from (A.10) that

$$
Q^{T} R^{T}=I \quad \text { and } \quad Q^{T} E=Q^{T} C^{T}=0 .
$$

Thus

$$
Q^{T} G_{m}^{-1} Q \Gamma_{m}=I
$$

proving (A) since $\Gamma_{m}$ is self adjoint.

(B) The claims for $\Gamma_{m}^{-1}$ follow immediately from (A), the positivity of $G_{m}^{-1}$ (A.7). Also, we get that $\Gamma_{m}^{-1}$ is bounded from above and hence $\Gamma_{m}>0$. We are thus left with establishing (A.9) for $\Gamma_{m}$. It is enough to prove the falloff for the operator

$$
\Gamma_{m}^{\prime}=G_{m}-G_{m} C^{T} G_{m+1}^{-1} C G_{m} .
$$

Explicit computation gives the momentum space representation of $\Gamma_{m x y}$ :

$$
\begin{aligned}
\Gamma_{m x y}= & \frac{1}{\left|\Lambda_{m}\right|}\left[\sum_{p}^{\prime} \hat{G}_{m}(p) e^{i p(x-y)}-\sum_{p}^{\prime \prime} \sum_{r: r_{\mu}=0, \pm 1} \frac{\hat{G}_{m}(p) \hat{G}_{m}\left(p+\frac{2 \pi r}{3}\right)}{3^{2} \hat{G}_{m+1}(3 p)}\right. \\
& \left.\cdot \prod_{\kappa}\left(\frac{1+2 \cos p_{\kappa}}{3} \cdot \frac{1+2 \cos \left(p_{\kappa}+\frac{2 \pi r_{k}}{3}\right)}{3}\right) e^{-i \frac{2 \pi}{3} r y} e^{i p(x-y)}\right],
\end{aligned}
$$

where $\sum_{p}^{\prime \prime}\left(\sum_{p}^{\prime \prime}\right)$ denotes the sum over

$$
0 \neq p \in 2 \pi 3^{-N+m} \mathbb{Z}^{d}, \quad\left|p_{\kappa}\right| \leqq \pi\left(p \in 3^{-N+m} \mathbb{Z}^{d}, \quad\left|p_{\kappa}\right| \leqq \pi, \quad 3 p \in 2 \pi \mathbb{Z}^{d}\right) .
$$

Consider the functions

$$
\begin{aligned}
\hat{\Gamma}_{m}(p, y):= & \hat{G}_{m}(p)-\sum_{r} \frac{\hat{G}_{m}(p) \hat{G}_{m}\left(p+\frac{2 \pi r}{3}\right)}{3^{2} \hat{G}_{m+1}(3 p)} \\
& \cdot \prod_{\kappa}\left(\frac{1+2 \cos p_{\kappa}}{3} \cdot \frac{1+2 \cos \left(p_{\kappa}+\frac{2 \pi r_{\kappa}}{3}\right)}{3}\right) e^{-i \frac{2 \pi}{3} r y}
\end{aligned}
$$

Lemma A.4. $\hat{\Gamma}_{m}(p, y)$ are analytic in $p_{0}$ and uniformly bounded on

$$
\left\{\left(p_{0}, \mathbf{p}\right) \in \mathbb{C} \times \mathbb{R}^{d-1}:\left|\operatorname{Im} p_{0}\right|<\varepsilon,\left|p_{i}\right| \leqq \pi\right\} .
$$


Proof. Again, given $\mathbf{p}$ and $y, \hat{\Gamma}_{m}(p, y)$ is meromorphic in $p_{0}$. To bound it uniformly we write

$$
\begin{gathered}
\hat{\Gamma}_{m}(p, y)=\frac{\hat{G}_{m}(p)}{3^{2} \hat{G}_{m+1}(3 p)}\left[3^{2} \hat{G}_{m+1}(3 p)-\hat{G}_{m}(p) \prod_{\kappa}\left(\frac{1+2 \cos p_{\kappa}}{2}\right)^{2}\right] \\
+\sum_{r \neq 0} \frac{\hat{G}_{m}(p) \hat{G}_{m}\left(p+\frac{2 \pi r}{3}\right)}{3^{2} \hat{G}_{m+1}(3 p)} \prod\left(\frac{1+2 \cos p_{\kappa}}{3} \frac{1+2 \cos \left(p_{\kappa}+\frac{2 \pi r_{\kappa}}{3}\right)}{3}\right) e^{-i \frac{2 \pi}{3} r y} .
\end{gathered}
$$

By (A.3)

$$
\begin{gathered}
3^{2} \hat{G}_{m+1}(3 p)-\hat{G}_{m}(p) \prod\left(\frac{1+2 \cos p_{\kappa}}{3}\right)^{2} \\
=\sum_{0 \neq r: r_{\kappa}=0, \pm 1} \hat{G}_{m}\left(p+\frac{2 \pi r}{3}\right) \prod\left(\frac{1+2 \cos \left(p_{\kappa}+\frac{2 \pi r_{\kappa}}{3}\right)}{3}\right)^{2}
\end{gathered}
$$

so that

$$
\begin{aligned}
\hat{\Gamma}_{m}(p, y)= & \sum_{r \neq 0} \frac{\hat{G}_{m}(p) \hat{G}_{m}\left(p+\frac{2 \pi r}{3}\right)}{3^{2} \hat{G}_{m+1}(3 p)} \Pi\left[\frac{1+2 \cos \left(p_{\kappa}+\frac{2 \pi r_{\kappa}}{3}\right)}{3}\right. \\
& \left.\cdot\left(\frac{1+2 \cos p_{\kappa}}{3} e^{-i \frac{2 \pi}{3} r y}+\frac{1+2 \cos \left(p_{\kappa}+\frac{2 \pi r_{\kappa}}{3}\right)}{3}\right)\right] .
\end{aligned}
$$

By Lemma A.1 $\frac{\hat{G}_{m}(p) \hat{G}_{m}\left(p+\frac{2 \pi r}{3}\right)}{G_{m+1}(3 p)}$ is uniformly bounded for $r \neq 0$ (poles of the numerator never coincide and get canceled by the zeros of the denominator).

From (A.13) we note that $\hat{\Gamma}_{m}(p, y)=\hat{G}_{m}(p)$ for $0 \neq 3 p \in 2 \pi \mathbb{Z}^{d}$ since $\hat{G}_{m+1}(3 p)^{-1}=0$. Also $\hat{\Gamma}_{m}(0, y)=0$ from (A.15) because $1+2 \cos \frac{ \pm 2 \pi}{3}=0$. So we can write

$$
\left(\Gamma_{m}^{\prime}\right)_{x y}=\frac{1}{\left|\Lambda_{m}\right|} \sum_{\substack{p \in 2 \pi 3^{-N}+m_{\mathbb{Z}^{d}} \\\left|p_{\mu}\right| \leqq \pi}} e^{i p(x-y)} \hat{\Gamma}_{m}(p, y)
$$

and the exponential falloff follows from Lemma A.4 as before.

Now we pass to the study of the operators $\mathscr{A}_{m}$ and $\nabla \mathscr{A}_{m}$. By explicit computation

$$
\begin{aligned}
\left(\mathscr{A}_{m}\right)_{z x}= & \frac{1}{\left|\Lambda_{m}\right|} \sum_{\substack{0 \neq p \in 2 \pi 3^{-N+m} \mathbb{Z}^{d} \\
\left|p_{\mu}\right| \leqq \pi}} e^{i p(z-y)} \sum_{M:\left|M_{\mu}\right| \leqq \frac{3^{m}}{2}} e^{2 \pi i M z}\left[G_{m}(p) \cdot 3^{2 m} \mu\left(\frac{p+2 \pi M}{3^{m}}\right)\right]^{-1} \\
& \cdot \prod_{\mu} \sin \frac{p_{\mu}+2 \pi M_{\mu}}{2}\left[3^{m} \sin \frac{p_{\mu}+2 \pi M_{\mu}}{2 \cdot 3^{m}}\right]^{-1} \\
\equiv & \frac{1}{\left|\Lambda_{m}\right|} \sum_{0 \neq p} \hat{A}_{m}(p, z) e^{i p(z-y)}
\end{aligned}
$$


and

$$
\begin{aligned}
\left(\nabla_{\mu} \mathscr{A}_{m}\right)_{z x}= & \left|\Lambda_{m}\right|^{-1} \sum_{\substack{p \in 2 \pi 3^{-N+m} \\
\left|p_{\kappa}\right| \leqq \pi}} e^{i p(z-x)} \sum_{M:\left|M_{\kappa}\right| \leqq \frac{3^{m}}{2}} e^{2 \pi i M z} \\
& \cdot\left[\hat{G}_{m}(p) \cdot 3^{2 m} \mu\left(\frac{p+2 \pi M}{3^{m}}\right)\right]^{-1} \cdot \prod_{\kappa} \sin \frac{p_{\kappa}+2 \pi M_{\kappa}}{2} \\
& \cdot 3^{m}\left(3^{m} \sin \frac{p_{\kappa}+2 \pi M_{\kappa}}{2 \cdot 3^{m}}\right)^{-1}\left(\exp i \frac{p_{\mu}+2 \pi M_{\mu}}{3^{m}}-1\right) \\
\equiv & \left|\Lambda_{m}\right|^{-1} \sum_{p} \widehat{\nabla_{\mu} A_{m}}(p, z) e^{i p(z-y)} .
\end{aligned}
$$

Lemma A.5. $\hat{\mathscr{A}}_{m}(p, z)$ and $\widehat{\nabla}_{\mu} \widehat{\mathscr{A}}_{m}(p, z)$ are analytic in $p_{0}$ and uniformly bounded when $\left|\operatorname{Im} p_{0}\right|<\varepsilon,\left|p_{k}\right| \leqq \pi$.

Proof. For fixed $\mathbf{p}$ and $z \hat{\mathscr{A}}_{m}$ and $\nabla_{\mu} \widehat{\mathscr{A}}$ are meromorphic in $p_{0}$, so it is enough to establish uniform bounds. Proceeding as in Lemma A.1:

$$
\left|\hat{\mathscr{A}}_{m}(p, z)\right| \leqq C\left|\frac{3^{-2 m} \mu\left(3^{-m} p\right)^{-1}}{G_{m}(p)}\right| \leqq C
$$

and similarly for $\widehat{\nabla}_{\mu}$

Again, Lemma A.5 leads to

Proposition A.6. There exist $C, \varepsilon>0$ such that the kernel

$$
\left(\mathscr{A}_{m}^{\prime}\right)_{z x}=\left|\Lambda_{m}\right|^{-1} \sum_{p \in 2 \pi 3-N+m_{\mathbb{Z}^{d}}} e^{i p(x-z)} \hat{\mathscr{A}}_{m}(p, z)
$$

satisfies

$$
\begin{aligned}
\left|\left(\mathscr{A}_{m}^{\prime}\right)_{z x}\right| & \leqq C e^{-\varepsilon|x-z|_{p}}, \\
\left|\left(\nabla \mathscr{A}_{m}^{\prime}\right)_{z x}\right| & \leqq C e^{-\varepsilon|x-z|_{p}} .
\end{aligned}
$$

Note that in $\mathscr{A}_{m}^{\prime}$ we also sum over the zero mode $p=0$ whereas in $\mathscr{A}_{m}$ this term is excluded. However, $\mathscr{A}_{m}$ and $\mathscr{A}_{m}^{\prime}$ coincide when acting on $\phi^{m}$ and $Q \xi^{m}$, both living in $(I-E) \mathbb{R}^{\Lambda_{m}}$. Propositions A.3 and A.6 give

Proposition A.7. There exist $C$ and $\varepsilon$ such that

$$
\left|\left\langle\nabla \zeta_{z}^{m} \nabla \zeta_{z^{\prime}}^{m}\right\rangle_{\Gamma_{m}}\right| \leqq C e^{-\varepsilon\left|z-z^{\prime}\right|_{p}} .
$$

Proof. We have by (2.32)

$$
\left\langle\nabla_{\mu} \zeta_{z}^{m} \nabla_{v} \zeta_{z^{\prime}}^{m}\right\rangle_{\Gamma_{m}}=\left(\nabla_{\mu} \mathscr{A}_{m} Q \Gamma_{m} Q^{+} \nabla_{v} \mathscr{A}_{m}\right)_{z z^{\prime}}
$$

and (A.20) is obvious by virtue of (A.9) and (A.19).

The last result concerns the decay of $\left\langle\nabla \psi_{z}^{m} \nabla \psi_{z^{\prime}}^{m}\right\rangle_{G_{m}}$.

Proposition A.8. There exist $C$ and $\varepsilon$ such that

$$
\left|\left\langle\nabla \psi_{z}^{m} \nabla \psi_{z^{\prime}}^{m}\right\rangle_{G_{m}}\right| \leqq C \frac{1}{1+\left|z-z^{\prime}\right|_{p}^{d}} .
$$


Proof. By iteration of (2.37) we obtain

$$
\begin{aligned}
\left\langle\nabla \psi_{z}^{m} \nabla \psi_{z^{\prime}}^{m}\right\rangle_{G_{m}}= & \left\langle\nabla \zeta_{z}^{m} \nabla \zeta_{z^{\prime}}^{m}\right\rangle_{\Gamma_{m}}+3^{-d}\left\langle\nabla \zeta_{\frac{z}{N-1}} \frac{\nabla \zeta^{N-1}}{\left.\frac{\bar{z}^{\prime}}{3^{N-1}}\right\rangle_{\Gamma_{N-1}}} .\right. \\
& +3^{-d(N-1)}\left\langle\nabla \zeta_{\left.\frac{N-\bar{z}}{3^{N-1}} \nabla \zeta \frac{\bar{z}^{\prime}}{3^{N-1}}\right\rangle_{\Gamma_{N-1}}} .\right.
\end{aligned}
$$

Hence, because of (A.20), (A.22) follows from the easy estimate

$$
\sum_{k=0}^{\infty} 3^{-d k} \exp -3^{-k} a \leqq C\left(1+a^{d}\right)^{-1}
$$

Acknowledgements. We are grateful to Professor J. Lebowitz for suggesting the problem and to Professor A. Jaffe and Dr. P. Collet for valuable discussions. One of the authors (K.G.) would like to thank Professor T. Balaban for conversations during which he learned about possibilities to use renormalization group as a rigorous tool in quantum field theory and statistical mechanics.

\section{References}

1. Wilson, K.G., Kogut, J.B.: Phys. Rep. 12 C, 75 (1974);

Domb, D., Green, M.S.: Phase transitions and critical phenomena, Vol. 6. New York: Academic Press 1976;

Ma, S.: Modern theory of critical phenomena. New York: Benjamin 1976;

Wallace, D.J., Zia, R.K.P.: Rep. Prog. Phys. 41, 1 (1978)

2. Bleher, P.M., Sinai, Ja.G.: Commun. Math. Phys. 33, 23 (1973);

Collet, P., Eckmann, J.-P.: A renormalization group analysis of the hierarcial model in statistical physics. Lecture Notes in Physics 74. Berlin, Heidelberg, New York: Springer 1978;

Sinai, Ja.G.: Proceedings of International Conference on Mathematical Physics in Rome 1977. Berlin, Heidelberg, New York: Springer 1977

3. Gallavotti, G.: Ann. Mat. Pure Appl. C XX, 1-23 (1978);

Benfatto, G., et al. : Commun. Math. Phys. 59, 143 (1978)

4. Benfatto, G., et al. : Commun. Math. Phys. 71, 95-130 (1980)

5. Balaban, T.: Proc. of the International Conference on Mathematical Physics, Lausanne 1979. Berlin, Heidelberg, New York: Springer 1980

6. Ruelle, D.: Commun. Math. Phys. 50, 189 (1976)

7. Kadanoff, L.P.: Physics 2, 263 (1965)

Communicated by A. Jaffe

Received May 5, 1980 